\title{
A new free-floating planet in the Upper Scorpius association
}

\author{
K. Peña Ramírez ${ }^{1,2}$, V. J. S. Béjar ${ }^{3,4}$, and M. R. Zapatero Osorio ${ }^{5}$ \\ ${ }^{1}$ Instituto de Astrofísica. Pontificia Universidad Católica de Chile (IA-PUC), 7820436 Santiago, Chile \\ e-mail: kpena@astro.puc.cl \\ 2 Millennium Institute of Astrophysics, Casilla 360, Santiago, Chile \\ 3 Instituto de Astrofísica de Canarias (IAC), 38205 La Laguna, Tenerife, Spain \\ ${ }^{4}$ Universidad de La Laguna, 38207 Tenerife, Spain \\ 5 Centro de Astrobiología (CSIC-INTA), 28850 Torrejón de Ardoz, Madrid, Spain
}

Received 22 September 2015 / Accepted 17 November 2015

\section{ABSTRACT}

\begin{abstract}
We report on a deep photometric survey covering an area of $1.17 \mathrm{deg}^{2}$ in the young Upper Scorpius stellar association using VIMOS $I z$ and UKIDSS ZJHK data that was taken with several years in between. The search for the least massive population of Upper Scorpius ( 5-10 Myr, $145 \mathrm{pc})$ is performed on the basis of various optical and infrared color-color and color-magnitude diagrams, including WISE photometry, in the magnitude interval $J=14.5-19 \mathrm{mag}$ (completeness), which corresponds to substellar masses from 0.028 through $0.004 M_{\odot}$ at the age and distance of Upper Scorpius. We also present the proper motion analysis of the photometric candidates, finding that two objects successfully pass all photometric and astrometric criteria for membership in the young stellar association. One of them, UScoJ155150.2-213457, is a new discovery. We obtained low resolution, near-infrared spectroscopy $(R \sim 450,0.85-2.35 \mu \mathrm{m})$ of this new finding using the FIRE instrument. We confirmed its low-gravity atmosphere expected for an Upper Scorpius member (weak alkaline lines, strong VO absorption, peaked $H$-band pseudocontinuum). By comparison with spectroscopic standards, we derive a spectral type of L6 \pm 1 , and estimate a mass of $\approx 0.008-0.010 M_{\odot}$ for USco J155150.2-213457. The colors and spectral slope of this object resemble those of other young, cool members of Upper Scorpius and $\sigma$ Orionis ( 3 Myr) and field, high gravity dwarfs of related classification in contrast with the very red indices of field, low gravity, L-type dwarfs of intermediate age. USco J155150.2-213457, which does not show infrared flux excesses up to $4.5 \mu \mathrm{m}$, becomes one of the least massive and latest type objects known in the entire Upper Scorpius stellar association.
\end{abstract}

Key words. open clusters and associations: individual: Upper Scorpius - brown dwarfs - stars: low-mass

\section{Introduction}

The shape of the initial brown dwarf and planetary mass function and the minimum mass for the collapse and fragmentation of clouds are crucial topics to be able to understand the dominant substellar formation process. Since substellar objects are significantly brighter and warmer at very young ages, e.g., less than 10 Myr (Chabrier et al. 2000), the detection of sources with a few Jupiter masses is possible by exploring nearby star-forming regions. Deep searches for the least massive population of these regions may shed light on the aforementioned topics.

The proximity and youth of the Upper Scorpius association (USco) make this region more than suitable for performing searches for members in the substellar regime. The entire USco covers a vast area of more than $200 \mathrm{deg}^{2}$ in the sky (Slesnick et al. 2008) and is located at a mean distance of $145 \pm 2 \mathrm{pc}$ (de Zeeuw et al. 1999). We carried out a deep photometric survey in a zone of low foreground extinction $\left(A_{v}<1 \mathrm{mag}\right.$; Preibisch et al. 1998, and references therein). Regarding the USco age, previous studies have suggested that the association is $\sim 5 \mathrm{Myr}$ old, based on the location of B-type stars in the Hertzsprung-Russell diagram and their comparison with evolutionary tracks (de Geus et al. 1989; Preibisch \& Zinnecker 1999; Preibisch et al. 2002). Recently, Herczeg \& Hillenbrand (2015) estimate an age of $\sim 4 \mathrm{Myr}$ for the low-mass members of the USco association, calculated from models that reproduce the lithium depletion boundary of various young star clusters and stellar moving groups. This is somehow younger than the USco age derived by Pecaut et al. (2012). These authors estimate an age of $11 \pm 2 \mathrm{Myr}$ for intermediate and high mass
USco members, including the M-type supergiant star Antares. The newly discovered eclipsing binaries in USco (UScoCTIO 5 and EPIC 203868608, Kraus et al. 2015; David et al. 2016) also support ages close to the $10 \mathrm{Myr}$ for this stellar association. In addition, the high proper motion of the USco association $\left(\mu=26.7 \pm 2.5{\text { mas } y r^{-1}}\right.$, Zacharias et al. 2004) is beneficial for the unambiguous identification of its true members once the astrometric and photometric studies are suitably combined.

Here, we present an exploration that extends over $1.17 \mathrm{deg}^{2}$ in the USco association. We used deep photometric data, which covers the wavelength range $0.8-3.4 \mu \mathrm{m}$. Our goal was to define the USco sequence of members with masses ranging from $\sim 0.025$ through $0.004 M_{\odot}$ within completeness. We also performed the spectroscopic follow-up of the faintest and least massive candidate found in our survey. The observational dataset is described in Sect. 2. The photometric and astrometric selection of USco member candidates and a discussion on possible field contaminants appear in Sect. 3. Section 4 introduces the spectroscopic data analysis of our faintest candidate. Mass values estimated from theoretical isochrones are presented in Sect.5.1. In Sect. 5.2 we place our search within the context of other explorations carried out in the USco region, and we discuss the implications of our findings for understanding the USco mass function. Our conclusions are given in Sect. 6.

\section{Observational data}

\subsection{Optical and near-infrared imaging}

Our survey, which covered an area of $1.17 \mathrm{deg}^{2}$ in USco, was carried out with the VIsible MultiObject Spectrograph 


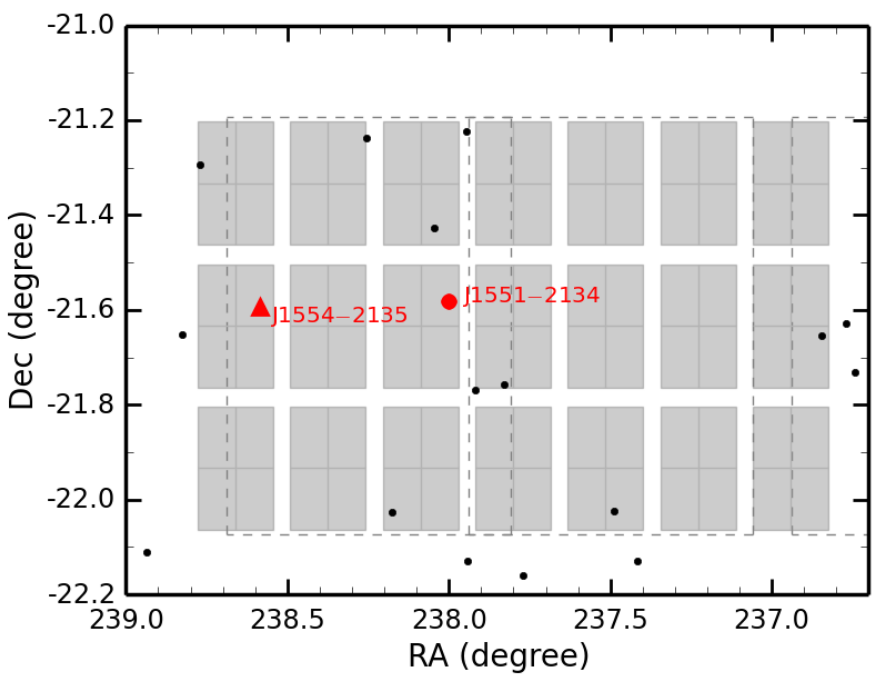

Fig. 1. Distribution of the 21 VIMOS pointings (gray squares) in the right ascension-declination diagram. The UKIDSS coverage overlapping with the VIMOS survey is indicated by the dashed gray lines (Lodieu et al. 2006, 2011). The red symbols represent the two final USco member candidates - labelled with their abridged names - selected from our combined photometric and astrometric study. Black dots represent the known USco member candidates and confirmed members (Lodieu et al. 2006, 2011; Béjar et al. 2009; Dawson et al. 2014) that fall within the VIMOS region and its surroundings.

(VIMOS; D'Odorico et al. 2003) installed on the Nasmyth A focus of 8-m Unit 3 (Melipal) of the Very Large Telescope (VLT) array sited on Cerro Paranal, Chile. In imaging mode, the VIMOS field of view is $7 \times 8 \mathrm{arcmin}^{2}$ per pointing. It uses a squared mosaic of four detectors $\left(2048 \times 2440\right.$ pix $\left.^{2}\right)$ separated by a gap of $2^{\prime}$. The instrumental pixel scale is 0.205 . A total of 21 VIMOS pointings were acquired using the $I$ and $z$ filters $^{1}$ and were designed to overlap with the area explored by the UKIRT Infrared Deep Sky Survey ${ }^{2}$ (UKIDSS, Lawrence et al. 2007). This gave us access to complementary photometric data in the ZYJHK bands, i.e., from 0.823 through $2.2 \mu \mathrm{m}$. Figure 1 shows the distribution of our VIMOS pointings in the north-east part of the USco region. Each pointing consisted of three exposures per filter with individual integration times of $160 \mathrm{~s}(I)$ and $105 \mathrm{~s}(z)$. The total exposure times were $8 \mathrm{~min}(I)$ and $5.2 \mathrm{~min}(z)$ per pointing. In Table 1, we provide the central coordinates of all VIMOS pointings, the observing filters and dates, the exposure times, and the average seeing conditions, as measured from the final reduced images.

The four detectors of VIMOS were treated separately for a proper processing of the raw data. Raw images were reduced using the CCDRED routine within the $\mathrm{IRAF}^{3}$ environment. We

\footnotetext{
1 Central wavelengths and bandwidths $(\mu \mathrm{m})$ as follows: $I_{\mathrm{c}}=0.823$, $z_{\mathrm{c}}=0.914 ; \Delta I=0.211, \Delta z=0.185$. The transmission of the VIMOS filters changes slightly from detector to detector. The values above correspond to the average of the four detectors. In this paper, all quoted magnitudes are given in the Vega system.

2 UKIDSS uses the UKIRT Wide Field Camera (WFCAM; Casali et al. (2007)) and a photometric system described in Hewett et al. (2006). The pipeline processing and science archive are described in Hambly et al. (2008). The UKIDSS broadband ZJHK data are directly calibrated from 2MASS point sources (Hodgkin et al. 2009).

3 The Image Reduction and Analysis Facility (IRAF) is distributed by National Optical Astronomy Observatories, which is operated by the Association of Universities for research in Astronomy, Inc., under contract to the National Science Foundation.
}

Table 1. VIMOS observing log, completeness and limiting magnitudes.

\begin{tabular}{|c|c|c|c|c|c|c|c|}
\hline Pointing & $\begin{array}{l}\text { RA (J2000) Dec } \\
\left(\begin{array}{ll}\left(\begin{array}{ll}\mathrm{h} \\
\mathrm{s}\end{array}\right)\left({ }^{\circ},{ }^{\prime \prime}\right)\end{array}\right.\end{array}$ & Filter & Date & $\begin{array}{l}\text { Texp } \\
\text { (s) }\end{array}$ & $\begin{array}{l}\text { Seeing } \\
\left({ }^{\prime \prime}\right)\end{array}$ & $\begin{array}{l}\text { Comp. } \\
\text { (mag) }\end{array}$ & $\begin{array}{l}\text { Lim. } \\
(\mathrm{mag})\end{array}$ \\
\hline \multirow[t]{2}{*}{1} & \multirow{2}{*}{$15: 53: 30.0-21: 19: 59.9$} & $I$ & 2009 Apr. 02 & $3 \times 160$ & 0.57 & 23.1 & 23.8 \\
\hline & & $z$ & 2009 Apr. 02 & $3 \times 105$ & 0.53 & 22.8 & 23.5 \\
\hline \multirow[t]{2}{*}{2} & \multirow{2}{*}{$15: 54: 38.8-21: 55: 59.9$} & $I$ & 2009 Apr. 02 & $3 \times 160$ & 0.66 & 22.8 & 23.8 \\
\hline & & $z$ & 2009 Apr. 02 & $3 \times 105$ & 0.55 & 22.4 & 23.3 \\
\hline \multirow[t]{2}{*}{3} & \multirow[t]{2}{*}{$15: 54: 38.8-21: 37: 59.9$} & I & 2009 Apr. 02 & $3 \times 160$ & 0.64 & 22.7 & 23.6 \\
\hline & & $z$ & 2009 Apr. 02 & $3 \times 105$ & 0.61 & 22.4 & 23.1 \\
\hline \multirow[t]{2}{*}{4} & \multirow{2}{*}{$15: 54: 38.8-21: 19: 59.9$} & $I$ & 2009 Apr. 02 & $3 \times 160$ & 0.58 & 23.0 & 23.9 \\
\hline & & $z$ & 2009 Apr. 02 & $3 \times 105$ & 0.58 & 23.0 & 23.7 \\
\hline \multirow[t]{2}{*}{5} & \multirow[t]{2}{*}{$15: 47: 46.0-21: 55: 59.9$} & $I$ & 2009 Apr. 20 & $3 \times 160$ & 0.86 & 22.0 & 23.0 \\
\hline & & $z$ & 2009 Apr. 20 & $3 \times 105$ & 0.78 & 21.7 & 22.6 \\
\hline \multirow[t]{2}{*}{6} & \multirow[t]{2}{*}{$15: 47: 46.0-21: 37: 59.9$} & $I$ & 2009 Apr. 20 & $3 \times 160$ & 0.73 & 22.5 & 23.3 \\
\hline & & $z$ & 2009 Apr. 20 & $3 \times 105$ & 0.63 & 22.1 & 23.1 \\
\hline \multirow[t]{2}{*}{7} & \multirow[t]{2}{*}{$15: 47: 46.0-21: 19: 59.9$} & $I$ & 2009 Apr. 20 & $3 \times 160$ & 0.79 & 22.3 & 23.2 \\
\hline & & $z$ & 2009 Apr. 20 & $3 \times 105$ & 0.74 & 22.0 & 22.7 \\
\hline \multirow[t]{2}{*}{8} & \multirow[t]{2}{*}{$15: 48: 54.7-21: 55: 59.9$} & $I$ & 2009 Apr. 20 & $3 \times 160$ & 0.66 & 22.4 & 23.7 \\
\hline & & $z$ & 2009 Apr. 20 & $3 \times 105$ & 0.56 & 22.3 & 23.2 \\
\hline \multirow[t]{2}{*}{9} & \multirow[t]{2}{*}{$15: 48: 54.7-21: 37: 59.9$} & $I$ & 2009 Apr. 20 & $3 \times 160$ & 0.63 & 22.0 & 23.3 \\
\hline & & $z$ & 2009 Apr. 20 & $3 \times 105$ & 0.66 & 21.9 & 22.6 \\
\hline \multirow[t]{2}{*}{10} & \multirow[t]{2}{*}{$15: 48: 54.7-21: 19: 59.9$} & $I$ & 2009 Apr. 20 & $3 \times 160$ & 0.66 & 22.6 & 23.5 \\
\hline & & $z$ & 2009 Apr. 20 & $3 \times 105$ & 0.66 & 22.2 & 23.0 \\
\hline \multirow[t]{2}{*}{11} & \multirow[t]{2}{*}{$15: 51: 12.4-21: 37: 59.9$} & $I$ & 2009 Apr. 21 & $3 \times 160$ & 0.53 & 23.3 & 24.1 \\
\hline & & $z$ & 2009 Apr. 21 & $3 \times 105$ & 0.50 & 22.8 & 23.8 \\
\hline \multirow[t]{2}{*}{12} & \multirow[t]{2}{*}{ 15:51:12.4-21:19:59.9 } & $I$ & 2009 Apr. 21 & $3 \times 160$ & 0.52 & 23.2 & 24.1 \\
\hline & & $z$ & 2009 Apr. 21 & $3 \times 105$ & 0.55 & 22.9 & 23.5 \\
\hline 13 & $15: 52: 21.1-21: 37: 59.9$ & $I$ & 2009 Apr. 21 & $3 \times 160$ & 0.68 & 22.8 & 23.6 \\
\hline & & $z$ & 2009 Apr. 21 & $3 \times 105$ & 0.59 & 22.4 & 23.2 \\
\hline 14 & $15: 52: 21.1-21: 55: 59.9$ & $I$ & 2009 Apr. 22 & $3 \times 160$ & 0.65 & 23.1 & 23.9 \\
\hline & & $z$ & 2009 Apr. 22 & $3 \times 105$ & 0.60 & 22.6 & 23.3 \\
\hline 15 & $15: 52: 21.1-21: 19: 59.9$ & $I$ & 2009 Apr. 22 & $3 \times 160$ & 0.52 & 23.2 & 24.0 \\
\hline & & $z$ & 2009 Apr. 22 & $3 \times 105$ & 0.54 & 22.7 & 23.4 \\
\hline 16 & $15: 53: 30.0-21: 55: 59.9$ & $I$ & 2009 Apr. 22 & $3 \times 160$ & 0.54 & 23.1 & 24.0 \\
\hline & & $z$ & 2009 Apr. 22 & $3 \times 105$ & 0.48 & 22.9 & 23.4 \\
\hline 17 & $15: 53: 30.0-21: 37: 59.9$ & $I$ & 2009 Apr. 22 & $3 \times 160$ & 0.53 & 23.0 & 23.9 \\
\hline & & $z$ & 2009 Apr. 22 & $3 \times 105$ & 0.49 & 22.8 & 23.4 \\
\hline 18 & $15: 50: 03.6-21: 55: 59.9$ & $I$ & 2009 Apr. 22 & $3 \times 160$ & 0.71 & 22.7 & 23.6 \\
\hline & & $z$ & 2009 Apr. 22 & $3 \times 105$ & 0.66 & 22.2 & 22.9 \\
\hline 19 & $15: 50: 03.6-21: 37: 59.9$ & $I$ & 2009 Apr. 22 & $3 \times 160$ & 0.64 & 22.8 & 23.7 \\
\hline & & $z$ & 2009 Apr. 22 & $3 \times 105$ & 0.54 & 22.6 & 23.1 \\
\hline 20 & $15: 50: 03.6-21: 19: 59.9$ & $I$ & 2009 Apr. 22 & $3 \times 160$ & 0.55 & 22.8 & 23.7 \\
\hline & & $z$ & 2009 Apr. 22 & $3 \times 105$ & 0.60 & 22.3 & 23.0 \\
\hline 21 & $15: 51: 12.4-21: 55: 59.9$ & $I$ & 2009 Apr. 22 & $3 \times 160$ & 0.60 & 22.6 & 23.5 \\
\hline & & $z$ & 2009 Apr. 22 & $3 \times 105$ & 0.56 & 22.4 & 23.0 \\
\hline
\end{tabular}

applied a bias correction using the overscan regions. The flatfield correction was done by generating a super-flat image (one per filter) from the median combination of all VIMOS science images. With these super-flat frames, we managed to reduce the fringing pattern, which is particularly strong for the $z$ band (Lagerholm et al. 2012). All science images were aligned and combined to produce deep frames per pointing and filter. Finally, we cut the vignetted areas from the processed images ${ }^{4}$. The area of $1.17 \mathrm{deg}^{2}$ was determined from the cut images.

The photometric analysis was made using DAOPHOT routines within IRAF. First, we automatically identified unresolved sources in the reduced images using the DAOFIND routine. Aperture and point-spread-function (PSF) photometry was performed on the identified sources with an aperture radius of $4 \times$ full width at half maximum (FWHM). The stellar PSF was defined by the Gaussian fit to about 10-15 isolated stars that were homogeneously distributed across the frames. Instrumental magnitudes in the $I$ band were converted into observed magnitudes using data from the DEep Near Infrared Survey of the Southern Sky (DENIS; Epchtein et al. 1994). We searched for common

4 In imaging mode, the VIMOS illuminated area is $\sim 1970 \times 2300$ pix $^{2}$. Exact values per detector were taken from VLT-MAN-ESO-14610-3509v86.1.pdf as of May 2015. 
sources between DENIS and our data in the DENIS magnitude range $I=17-18$ mag. Costado et al. (2005) find that the DENIS $I$ and VIMOS $I_{\text {Cousin }}$ magnitudes are quite alike with a negligible difference of $I_{\text {Cousin }}-I_{\text {DENIS }}=0.03 \pm 0.04$ mag for $\mathrm{M}$ - and L-type sources, which are the spectral-type domains of interest to us. Therefore, we did not correct the VIMOS I-band magnitudes for any color term. Regarding the $z$-band photometry, the VIMOS instrumental magnitudes were calibrated using the $Z$-band data from the UKIDSS Eight Data Release (DR8), using sources in common that have UKIDSS magnitudes in the interval $Z=16-17.5 \mathrm{mag}$. The VIMOS $z$ filter is centered at a redder wavelength than the UKIDSS Z-band; however, after multiplying the VIMOS $z$ filter by the response function of the detectors, the bandpasses of the two filters become very similar. To further test whether there is a color term between VIMOS $z$ and UKIDSS $Z$ magnitudes, we retrieved the near-infrared spectra of L-type field dwarfs from the catalog by Cushing et al. (2005). All spectra were conveniently convolved with the filter transmission profiles and corresponding detectors' response functions and integrated to derive the $z$ - and $Z$-magnitudes. We found that the resulting magnitudes are alike with negligible differences, which confirms that no color term is required for calibrating the VIMOS $z$-band. The dispersion of the photometric calibration was determined at \pm 0.09 mag for the $I$ band and \pm 0.04 mag for the $z$ band. These quantities were quadratically added to the instrumental PSF magnitude errors provided by IRAF.

We estimated the completeness magnitudes of the VIMOS survey ( $I z$ bands) and the combined VIMOS-UKIDSS exploration ( $I z+Z J H K$ bands, see Sect. 3.1) by comparing the total number of identified sources per magnitude interval with an exponential distribution of stars. The exponential fit was obtained for each filter independently, using bright-to-intermediate magnitude sources. The completeness magnitude is defined as the magnitude interval that immediately precedes the magnitude bins that display a continuous deficit of stars with respect to the exponential prediction. The limiting magnitude is calculated as the magnitude bin at which the total number of sources deviates by $\geq 50 \%$ from the counts of the completeness magnitude bin. Our determined completeness and limiting magnitudes are given in Table 1 and roughly correspond to source detections around the $10 \sigma$ and $4 \sigma$ levels, respectively ( $\sigma$ is the sky background noise). In short, the USco VIMOS survey is complete down to $z=21.7$ and $I=22.0 \mathrm{mag}$. These values correspond to the shallowest images of VIMOS pointing Number 5 , whose source counts versus observed magnitudes are illustrated in Fig. 2. The deepest VIMOS pointing (Number 11) has images that are $\sim 1.1 \mathrm{mag}$ fainter in both bands. The limiting magnitudes of the VIMOS survey are $z=23.5$ and $I=23.8$ mag (computed as the mean values of all 21 pointings). Regarding the UKIDSS data and following the same approach, we determined the following completeness and limiting magnitudes: $Z=20.8$, $Y=20.5, J=19.0, H=18.7$, and $K=18.0 \mathrm{mag}$ (completeness), and $Z=21.5, Y=21.2, J=20.0, H=19.4$, and $K=18.7 \mathrm{mag}$ (limiting magnitudes). These quantities agree with the values given by other groups (Lodieu et al. 2006) and with the information provided on the UKIDSS webpage ${ }^{5}$. In view of these numbers, our VIMOS survey is two magnitudes deeper than the UKIDSS data.

The VIMOS data were astrometrically calibrated using the right ascension and declination coordinates of the 2MASS

\footnotetext{
5 http://surveys.roe.ac.uk/wsa/dr8plus_release.html as of July 2015.
}

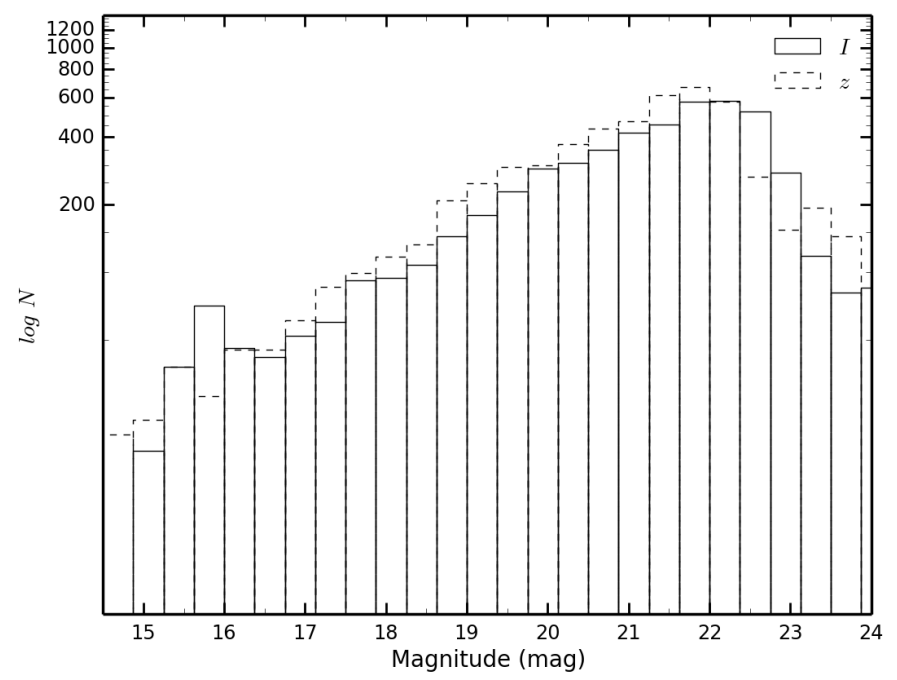

Fig. 2. Source counts as a function of observed VIMOS $I$ and $z$ magnitudes for the fifth pointing, which has the shallowest images and defines the completeness magnitudes of the $I z$ survey. The bin size is 0.4 mag.

catalog (Skrutskie et al. 2006). The internal precision of the astrometric calibration is \pm 0.2 .

\subsection{Near-infrared spectroscopy}

Near-infrared JHK spectroscopy of USco J155150.2-213457 (hereafter J1551-2134, see Sect. 3) was obtained using the Folded-port InfraRed Echellette (FIRE; Simcoe et al. 2008, 2013) instrument installed at the 6.5-m Baade Telescope, one of the Magellan telescopes at Las Campanas Observatory (Chile). FIRE is a near-infrared dual-mode spectrometer (detector pixel size of 0.15 ) that operates over the $0.82-2.51 \mu \mathrm{m}$ wavelength range. We used the FIRE high-throughput prism mode with a slit width of $0 . ' 6$. This instrumental configuration yielded a spectral nominal dispersion of $9.25 \AA \mathrm{pix}^{-1}$ and a resolving power of about 450 at the central frequency $\left(\lambda_{\mathrm{c}} \sim 1.66 \mu \mathrm{m}\right)$. Because of the faint nature of our target, the useful wavelength spectrum coverage is $0.85-2.35 \mu \mathrm{m}$. Observations of J1551-2134 were collected with a seeing of 0.5-0.'6 on 2015 May 13.

J1551-2134 and a bright reference star $(J=14.6 \mathrm{mag})$ at a separation of 29.6 from the science target were acquired with the $J$-band filter and simultaneously aligned on the nominal 1'-length slit. Individual $300 \mathrm{~s}$ exposures were obtained with the source at two nod positions separated by $5{ }^{\prime \prime} 0$. Both the science target and the reference star were twice observed in an ABBA nodding pattern, yielding a total on-source integration time of $40 \mathrm{~min}$. A standard A0-type star was observed immediately after the science observations with a similar airmass to ensure a proper correction for the telluric contribution. Reduction of the raw data was accomplished using IRAF routines. The $\mathrm{AB}$ nodded frames were subtracted to remove the background emission contribution. Individual sky-subtracted frames were then registered using the bright reference star and stacked together to produce one high signal-to-noise $(\mathrm{S} / \mathrm{N})$ image. The spectra of J1551-2134 and the telluric standard star were optimally extracted and wavelength-calibrated using a NeNeAr lamp exposure. After removal of the intrinsic features (typically hydrogen lines) of the A0-type star, the calibration spectrum was divided into the corresponding target data to remove telluric absorptions and instrumental spectral response. Finally, the data 
were multiplied by a blackbody curve of $9700 \mathrm{~K}$ to restore the spectral slope of J1551-2134.

\section{Selection of USco member candidates}

\subsection{VIMOS-UKIDSS zJ search}

Given the depth of the entire photometric datasets (VIMOS and UKIDSS) available to us and the fact that we were searching for faint sources with red optical to near-infrared colors, we concluded that the combined VIMOS $z$ - and UKIDSS $J$-band survey may yield promising USco member candidates. We retrieved UKIDSS DR8 $J$-band photometry for the sources identified in our VIMOS data using a correlation radius of 2 '. 0 . Sometimes, two VIMOS sources that are close to each other and have very different brightness were cross-correlated with one single, typically bright UKIDSS source (wrongly identified as a probable galaxy by the UKIDSS algorithms in many cases), possibly because of the differing spatial scale and depth of the two catalogs. This resulted in false red $z-J$ colors that may have contaminated the $z J$ color-magnitude diagram. To prevent this from happening, we discarded the VIMOS faint objects with bright sources at less than 2'. 0 . Nevertheless, we first secured that none of them had red VIMOS $I-z$ colors in the region of interest, as defined in Sect. 3.2.

We built the $z J$ color-magnitude diagram depicted in Fig. 3. The dynamic range of the VIMOS-UKIDSS survey covers $5.5 \mathrm{mag}$ from $J=14.5$ through $20.0 \mathrm{mag}$. The faint end is imposed by the UKIDSS limiting magnitude and the bright limit is such that it avoids the highly saturated sources and sources with magnitudes in the nonlinear regime of the detectors. The completeness of the $z J$ survey is given by the interval $J=14.5-$ $19.0 \mathrm{mag}$. As seen in Fig. 3, the USco photometric sequence is well delineated by known members of the association (plotted as filled blue dots). In the surveyed area and with magnitudes fainter than $J=14.5 \mathrm{mag}$, there are seven USco candidates that have been previously published in the literature. They were detected photometrically by Lodieu et al. (2006, 2007) and all have spectroscopic follow-up observations in Lodieu et al. $(2008,2011)$. These objects show colors, proper motions, $\mathrm{H} \alpha$ emission, and other spectroscopic features typical of lowgravity atmospheres, which support their membership in the USco association.

The separator that we used to discriminate USco member candidates from field sources in Fig. 3 is formed by two straight lines. One of these lines covers the interval $J=14.5-16.5 \mathrm{mag}$ and runs parallel to the USco sequence of known objects shifted by $\sim 4 \sigma$ towards blue $z-J$ colors, where $\sigma$ represents the color dispersion of the USco photometric sequence. The other line separator was employed for the interval $J=16.5-19.0 \mathrm{mag}$ and was based on the separator proposed by Lodieu et al. (2007): it goes from $(z-J, J)=(1.7,16.5)$ to $(2.0,19.0) \mathrm{mag}$. The defined field-USco separator is shown with a solid line in Fig. 3. USco member candidates must fall to the red of the separator line. This is a rather conservative selection criterion that is intended to identify all possible candidates. We acknowledged that additional criteria were required to clean the list of photometric candidates and avoid contaminants.

Within the VIMOS-UKIDSS survey completeness $(J=$ 14.5-19 mag), a total of 92 sources were found to be populating the red side of the defined separator in Fig. 3. Of them, 65 are resolved (their FWHMs are at least 1.5 times higher than the width provided by the average seeing), and 27 sources appear to be point-like objects. We did not consider the 65 re-

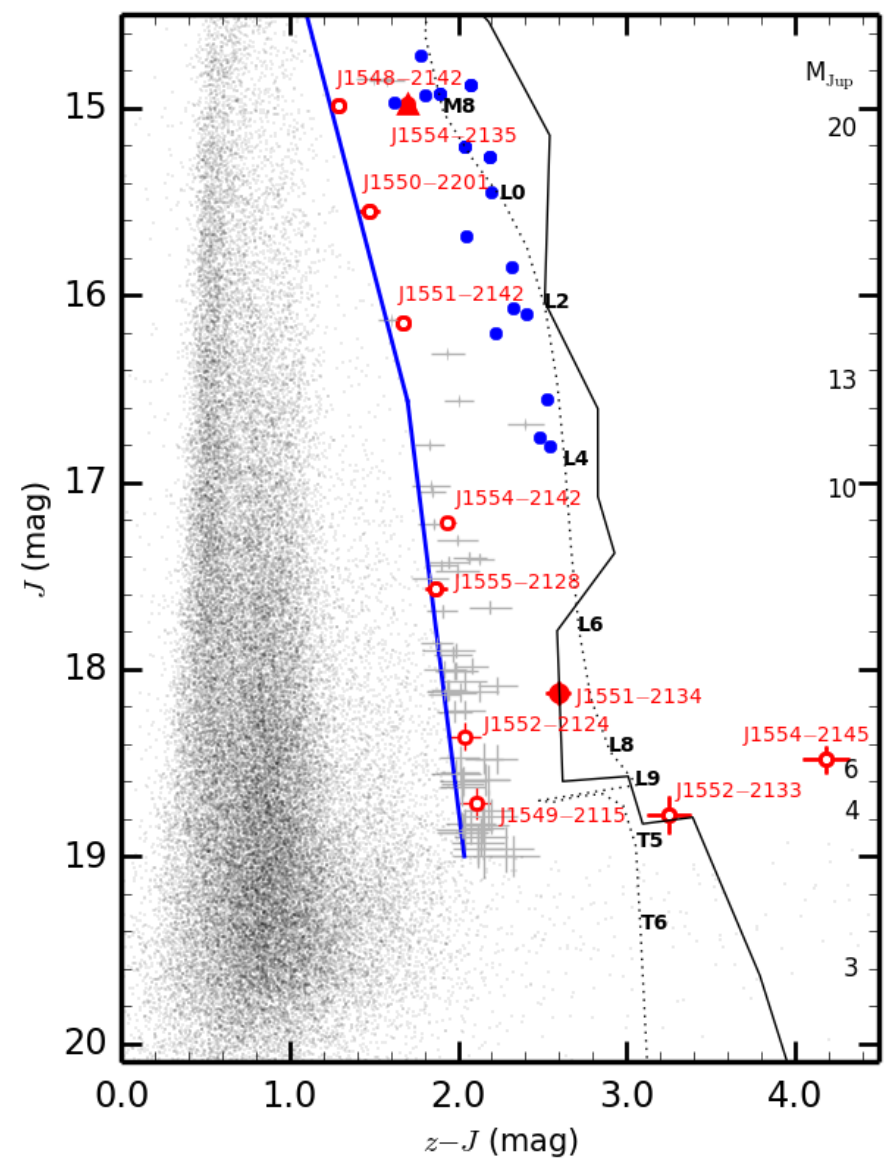

Fig. 3. Color-magnitude diagram, $J$ versus $z-J$, of the VIMOSUKIDSS search. Previously known USco members are shown with blue filled dots. The separator (two blue straight lines) used for the selection of USco member candidates goes from $J=14.5$ to $19.0 \mathrm{mag}$. The 11 unresolved sources - labeled with their abridged names - that pass the $z J$ criterion are plotted as red symbols: the new candidate is shown with a red solid dot, while the previously known USco member recovered here, USco J155419.99-213543.1 (Lodieu et al. 2006, 2008), is depicted with a red-filled triangle. The resolved sources complying with our $z J$ criterion are plotted as gray crosses, and the bulk of field detections are shown with small black dots. For comparison purposes, the average sequence of field M-, L-, and T-dwarfs normalized to the midM members of USco (not shown) is over-plotted with a dotted line. The 5-Myr isochrone of Chabrier et al. (2000) is included with a solid line (we adopted a distance of $145 \mathrm{pc}$ ). Its corresponding masses (Jovian units) are indicated on the right side.

solved objects in our list of USco candidates. We checked the catalogued aperture UKIDSS photometry by obtaining the PSF photometry of the 27 unresolved objects, the majority of which are quite faint and close to the completeness magnitude of the survey. The new PSF photometry located 16 out of 27 sources in the blue of our $z J$ selection criterion (many of these objects have aperture photometry contaminated by nearby bright stars), and they were rejected. Only 11 unresolved sources remained as $z J$ USco member candidates. These are depicted and labelled with their abridged names in Fig. 3. In Table 2, we provide their photometry. With the exception of three objects, most lie rather close to the artificial boundary that was defined to separate USco candidates from field sources.

Of the 11 USco candidates, two were previously known: USco J155419.99-213543.1 (J1554-2135) and USco J155011.5-220122 (2MASS J15501151-2201213). The former object was first identified from an UKIDSS-only survey 
Table 2. USco member candidates selected from the combined VIMOS-UKIDSS $z J$ search.

\begin{tabular}{|c|c|c|c|c|c|c|c|c|c|c|}
\hline $\begin{array}{l}\text { RA (J2000) Dec } \\
\left(\begin{array}{lll}(\mathrm{h} \mathrm{m} & \mathrm{s}\end{array}\right)\left({ }^{\circ},{ }^{\prime}\right)\end{array}$ & Name & $\begin{array}{c}I \\
(\mathrm{mag}) \\
\end{array}$ & $\begin{array}{c}z \\
(\mathrm{mag}) \\
\end{array}$ & $\begin{array}{c}Z \\
(\mathrm{mag}) \\
\end{array}$ & $\begin{array}{c}Y \\
(\mathrm{mag})\end{array}$ & $\begin{array}{c}J \\
(\mathrm{mag}) \\
\end{array}$ & $\begin{array}{c}H \\
(\mathrm{mag}) \\
\end{array}$ & $\begin{array}{c}K \\
(\mathrm{mag}) \\
\end{array}$ & $\begin{array}{c}W 1 \\
(\mathrm{mag})\end{array}$ & $\begin{array}{c}W 2 \\
(\mathrm{mag})\end{array}$ \\
\hline $155419.9-213543$ & USco J155419.9-213543 ${ }^{a}$ & $17.680 \pm 0.113$ & $16.670 \pm 0.026$ & $16.852 \pm 0.009$ & $15.847 \pm 0.005$ & $14.975 \pm 0.004$ & $14.321 \pm 0.003$ & $13.746 \pm 0.003$ & $13.260 \pm 0.025$ & $12.584 \pm 0.027$ \\
\hline 214242 & UScc & 10 & 78 & & 55 & & & & 27 & 41 \\
\hline $155011.5-220122$ & USco J155011.5-220122 ${ }^{b}$ & $18.044 \pm 0.071$ & $17.018 \pm$ & $17.209 \pm$ & $16.337 \pm 0.008$ & $548 \pm 0.006$ & $14.979 \pm 0.006$ & $14.572 \pm 0.007$ & $14.397 \pm$ & $14.193=$ \\
\hline $155132.7-214243$ & USco J155132.7-214243 & $18.600 \pm 0.104$ & $17.823 \pm 0.034$ & $17.951 \pm 0.018$ & $17.000 \pm 0.011$ & $16.148 \pm 0.010$ & $15.593 \pm 0.009$ & 15.108 & 14.923 & 14.77 \\
\hline $155450.8-214232$ & USco J155450.8 & $20.313 \pm 0.122$ & $19.153 \pm$ & 8 & $56=$ & 24 & 16.506 & 15.966 & $15.790 \pm 0.059$ & $15.669 \pm 0.173$ \\
\hline $155508.4-212842$ & USco J155508. & $20.439 \pm$ & $19.435 \pm$ & $19.503 \pm$ & $481 \pm$ & $17.566=$ & 0 & 16.388 & & \\
\hline-213457 & USco J & $21.896 \pm$ & $20.724 \pm$ & $20.658 \pm 0.163$ & $19.608 \pm$ & 60 & 42 & 39 & $45 \pm 0$ & $61 \pm 0$ \\
\hline $154954.5-211504$ & USco J154954.5-211504 ${ }^{d}$ & $22.070 \pm 0.080$ & $20.821 \pm 0.042$ & $\cdots$ & $19.935 \pm 0.087$ & $18.715 \pm 0.083$ & $17.836 \pm 0.070$ & $17.278 \pm 0.077$ & $17.172 \pm 0.175$ & $15.864 \pm 0.207$ \\
\hline-213341 & co. & 22.485 & $22.027 \pm$ & 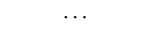 & $19.623 \pm$ & 18.778 & 18.853 & $\geq 1$ & $\cdots$ & $\cdots$ \\
\hline $155454.1-214555$ & USco J155454.1-214555 & $23.296 \pm 0.140$ & $22.669 \pm 0.118$ & $19.762 \pm 0.064$ & $19.315 \pm 0.066$ & $18.483 \pm 0.075$ & $18.203 \pm 0.105$ & $18.380 \pm 0.231$ & $\cdots$ & $\cdots$ \\
\hline
\end{tabular}

Notes. Right ascension (RA) and declination (Dec) coordinates as derived from the VIMOS astrometric calibration. Top and bottom panels list the 11 photometric USco candidates from the combined VIMOS-UKIDSS Survey (Sect. 3.1). The top panel indicates candidates that were also found in the VIMOS-only search (Sect. 3.2). ${ }^{(a)}$ USco member discovered and confirmed by Lodieu et al. (2006, 2008). It has $W 3=11.034 \pm 0.136$ mag. ${ }^{(b)}$ High proper motion source (Sect. 3.5) discovered by Deacon et al. (2009). ${ }^{(c)}$ Candidate with spectroscopic follow-up (Sect. 4). ${ }^{(d)}$ Nonphotometric candidate according to the diagrams of Fig. 5. ${ }^{(e)}$ We provided $J$-band PSF photometry. ZYHK photometry as in the UKIDSS catalog (we checked that the PSF photometry of these bands agrees with UKIDSS aperture photometry within the quoted error bars).

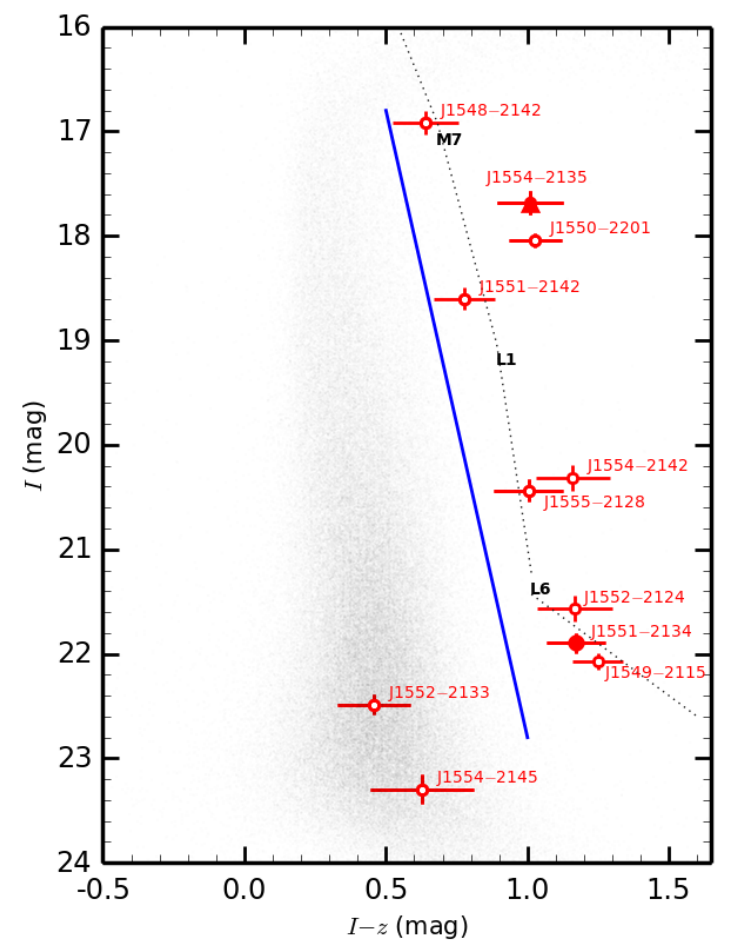

Fig. 4. Color-magnitude diagram, $I$ versus $I-z$, of the VIMOS-only search. Symbols as in Fig. 3. The solid blue line separates USco member candidates from other sources. The VIMOS-UKIDSS candidates J1552-2133 and J1554-2145 fail the $I z$ criterion for USco candidates.

by Lodieu et al. (2006). In Lodieu et al. (2008) it was confirmed as an M8.0 bonafide member of the USco association. The latter object was first reported to have a high proper motion from the cross-correlation of the UKIDSS and 2MASS catalogs by Deacon et al. (2009), and see Sect. 3.5. Except for these two sources, none of the USco candidates published by other groups has a magnitude brightness within the dynamic range or lies within the effective area of our study.

\subsection{VIMOS Iz selection}

To provide a more reliable list of USco member candidates, we combined the previous $z J$ search with a selection based only on
VIMOS data. We used the $I$-band versus $I-z$ color-magnitude diagram depicted in Fig. 4. Our criterion to select USco member candidates followed the prescription proposed by Ardila et al. (2000) for a similar diagram. These authors deal with candidates in the magnitude interval $I=13-19 \mathrm{mag}$, which includes the latetype $\mathrm{M}$ dwarfs. Our survey is deeper than theirs; we thus linearly extrapolated the proposed criterion towards fainter magnitudes and the L-types as shown by the solid line in Fig. 4. This extrapolation falls to the blue of the photometric sequence of field $\mathrm{L}$ dwarfs down to the completeness of the VIMOS data. The field sequence displayed in Figs. 3 and 4 was built by using data from Hewett et al. (2006), Patten et al. (2006), and Leggett et al. (2007), as described in Sect. 3.3.

Nine candidates from the VIMOS-UKIDSS $z J$ search were found to fulfil the $I z$ criterion, i.e., they lie to the red of the fieldcluster photometric separator. The nine sources are included in the top panel of Table 2. Only two $z J$ candidates failed the $I z$ selection process (bottom panel of Table 2) because they display blue $I-z$ colors despite having the faintest $I$ magnitudes in our list of candidates. Surprisingly, they were the two reddest candidates in the $z J$ search. Next, we investigated these two sources in detail.

To confirm the photometry of J1554-2145, we collected $J$-band images with the Long-slit Intermediate Resolution Infrared Spectrograph (LIRIS; Manchado et al. 2004) on the William Herschel Telescope (WHT) on 2012 June 15. LIRIS has a HAWAII detector of $1024 \times 1024$ pix $^{2}$ with a plate scale of 0 .' 25 per pixel. In imaging mode, LIRIS has a field of view of $4.27 \times 4.27 \operatorname{arcmin}^{2}$. The LIRIS image provided us with a time baseline of $7 \mathrm{yr}$ with respect to the older UKIDSS data, and of $5.2 \mathrm{yr}$ with respect to the VIMOS images. The LIRIS $J$-band data were acquired with a dithering pattern of nine positions over the detector; individual exposure time was $50 \mathrm{~s}$ per dither, and the total exposure time was $2250 \mathrm{~s}$. Observing conditions were photometric with a seeing of $1.4^{\prime \prime}$. Data reduction included flat field correction and sky subtraction using routines within the IRAF environment. Individual images were aligned and combined to produce deep data in the $J$ band. The final LIRIS image has a limiting magnitude of $J=20.7 \mathrm{mag}$ at the $4 \sigma$ level. This photon depth would have been sufficient to guarantee the detection of J1554-2145 with an excellent $\mathrm{S} / \mathrm{N}$ if the object's brightness had been that of the UKIDSS catalog $(J=$ $18.483 \mathrm{mag}$ ). However, this particular source was not detected 
in the LIRIS image, indicating that at the time of the LIRIS observations, J1554-2145 had $J \geq 20.7 \mathrm{mag}$, in high contrast with the UKIDSS $J$-band measurement. Based on this result, we concluded that J1554-2145 is a variable source, whose nature cannot be unveiled with our current data. It might actually be an USco member; there are known young sources with strong photometric variations due to circumbinary disks in Orion, or stars like KH 15D (Johnson et al. 2004) and CHS 7797 (RodríguezLedesma et al. 2012). To the best of our knowledge, J1554-2145 has not been previously identified in any variable or extragalactic source catalog or in supernovae databases.

The VIMOS $z$ and UKIDSS $J$ equatorial coordinates of J1552-2133 differ by 1 '. 5 . This suggests that either we identified two different sources at optical and near-infrared wavelengths, or

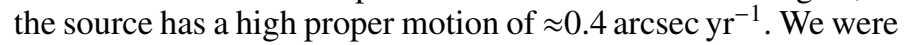
convinced that the VIMOS $z$ and $I$ identifications correspond to one source because both data were taken at the same time and both $z$ and $I$ coordinates agree within the astrometric error bars. We thus relied on the $I-z$ color. Unfortunately, we cannot confirm whether the UKIDSS detection corresponds to the VIMOS object. In any case, this source does not appear to be a member of USco according to our selection criteria: either its $I-z$ color is too blue or it has a high proper motion that is inconsistent with the stellar association. From now on, neither J1554-2145 nor J1552-2133 (bottom panel of Table 2) are considered as USco photometric candidates. Therefore, we were left with nine candidates (two of which are known in the literature) that successfully pass the $z J$ and $I z$ photometric criteria down to the completeness magnitude of the VIMOS-UKIDSS survey.

To fully exploit the combined VIMOS-UKIDSS survey between completeness and limiting magnitudes, we searched for sources with the following photometric criteria, which are valid to identifying cool dwarfs with spectral types later than earlyL in USco: $J>19.0, I>22.0, z-J>2$, and $I-z>1$ mag, or $I$-band non-detections. Ten objects were found with colors redder than the $z J$ field-USco boundary in Fig. 3, seven of which turned out to be false detections close to the spikes of very bright stars. One showed an extended profile with 3.2 times the stellar FWHM, and the remaining two unresolved sources lay near bright stars. We carried out their PSF photometry using the UKIDSS images and obtained that their new colors were not compatible with our criteria (they moved to the cloud of field objects, i.e., to the blue side of the separator in Fig. 3). In short, the search for new USco member candidates with VIMOS and UKIDSS magnitudes beyond completeness yielded no new objects of interest. Therefore, our USco candidates are those listed in the top panel of Table 2.

\subsection{Additional photometric criteria}

To provide further robustness to our list of USco member candidates, we built additional color-magnitude and color-color diagrams. Figure 5 depicts a variety of these diagramas using the VIMOS, UKIDSS, and WISE photometry, thus covering the wavelength interval between 0.8 and $3.4 \mu \mathrm{m}$. The nine candidates from the top panel of Table 2 are indicated with red symbols and are labelled in all panels of the figure. We also included the known USco confirmed members and photometric candidates published by Ardila et al. (2000), Lodieu et al. (2011), and Lodieu et al. (2013),

The $I-J$ versus $J-K$ color-color panel of Fig. 5 is useful for discriminating extragalactic sources from very red dwarfs (see the discussion by Bihain et al. 2009). In this panel, we added the resolved objects found in Sect. 3.1, i.e., whose FWHMs were
1.5 times higher than the stellar PSF. All of these objects occupy a particular region of the color-color diagram: they tend to have red $J-K$ values while their $I-J$ colors do not exceed the 3mag boundary. These colors, where the $K$ band is particularly red, are typical of galaxies (Franx et al. 2003). None of the nine USco photometric candidates fall within this region; on the contrary, they follow, and actually extrapolate, the color-color sequence that has been defined by the previously confirmed USco members. This adds consistency to our photometric selection of unresolved objects, as described in previous sections.

The USco candidates J1552-2124 and J1549-2115, which have the faintest $J$-band magnitudes in Table 2, display $J-H$, $J-K$, and $J-W 1$ colors that are bluer than other USco objects. As shown in Fig. 5, and in comparison with the field sequence, these two objects may have early-T spectral types if they were USco members and if the USco sequence were described by the field sequence of red dwarfs. The average location of field dwarfs between the mid-M types and the late-T types shown in Figs. 3-5 was constructed by calculating the mean magnitudes and colors for each spectral type (Hewett et al. 2006) and by using the equations published by Dupuy \& Liu (2012). It was normalized to the location of thirteen USco M5.5-type dwarfs taken from Slesnick et al. (2008). However, J1552-2124 and J1549-2115 do not display $I-z$ and $z-J$ colors that are fully compatible with T0-T5 dwarfs and their blue deviation from the trend, which is delineated by other USco candidates in the color-magnitude diagrams of Fig. 5, was interpreted as a likely non-membership signature.

From the combined 1.17- $\mathrm{deg}^{2}$ VIMOS-UKIDSS USco survey that explores the magnitude interval $J=14.5-19$ mag, we identified a total of seven photometric member candidates with colors that cover the broad $0.8-3.4 \mu \mathrm{m}$ wavelength range, which is consistent with membership in the young stellar association. They likely have spectral types between late-M and mid-L as can be inferred from a direct comparison of their colors with the indices of field, high-gravity dwarfs.

\subsection{Contamination}

Our photometric search may suffer from some contamination, which we study next. We expected the main source of contaminants to be Galactic field sources of M, L and T spectral types, and reddened galaxies. We derived the Galactic contamination contribution following the prescription given in Caballero et al. (2008, and references therein). In the range of magnitudes $J=14.5-19 \mathrm{mag}$ (the $z J$ search), a total of $\sim 4.5$ field M, $\mathrm{L}$, and $\mathrm{T}$ dwarfs are expected to pollute the explored regions of the color-magnitude diagrams of previous sections. These contaminant objects are likely interlopers, which are distributed in spectral types and magnitude intervals as follows: $\sim 2.7$ field M dwarfs would appear in the range $J=14.5-16.0 \mathrm{mag}$, and $\sim 1.6$ field $\mathrm{L}$ dwarfs could be contaminating the faintest magnitude bin $J=16-19 \mathrm{mag}$. As for the field $\mathrm{T}$ dwarfs, the expected pollution was small with roughly $\sim 0.2$ T-type objects at the faintest magnitudes of $J=18-19 \mathrm{mag}$. The contamination resulting from background giant red stars of $\mathrm{M}$ spectral types was expected to be negligible since the USco stellar association is located at latitudes above the Galactic plane $(b \sim+20 \mathrm{deg})$. The derived contamination by field cool dwarfs with spectral types similar to those we were seeking in USco suggested that, out of the seven photometric candidates, only $2-3$ would remain as USco members.

Regarding the extragalactic contamination, the good seeing of the VIMOS data and the small size of the VIMOS pixel 
K. Peña Ramírez et al.: A new free-floating planet in the Upper Scorpius association
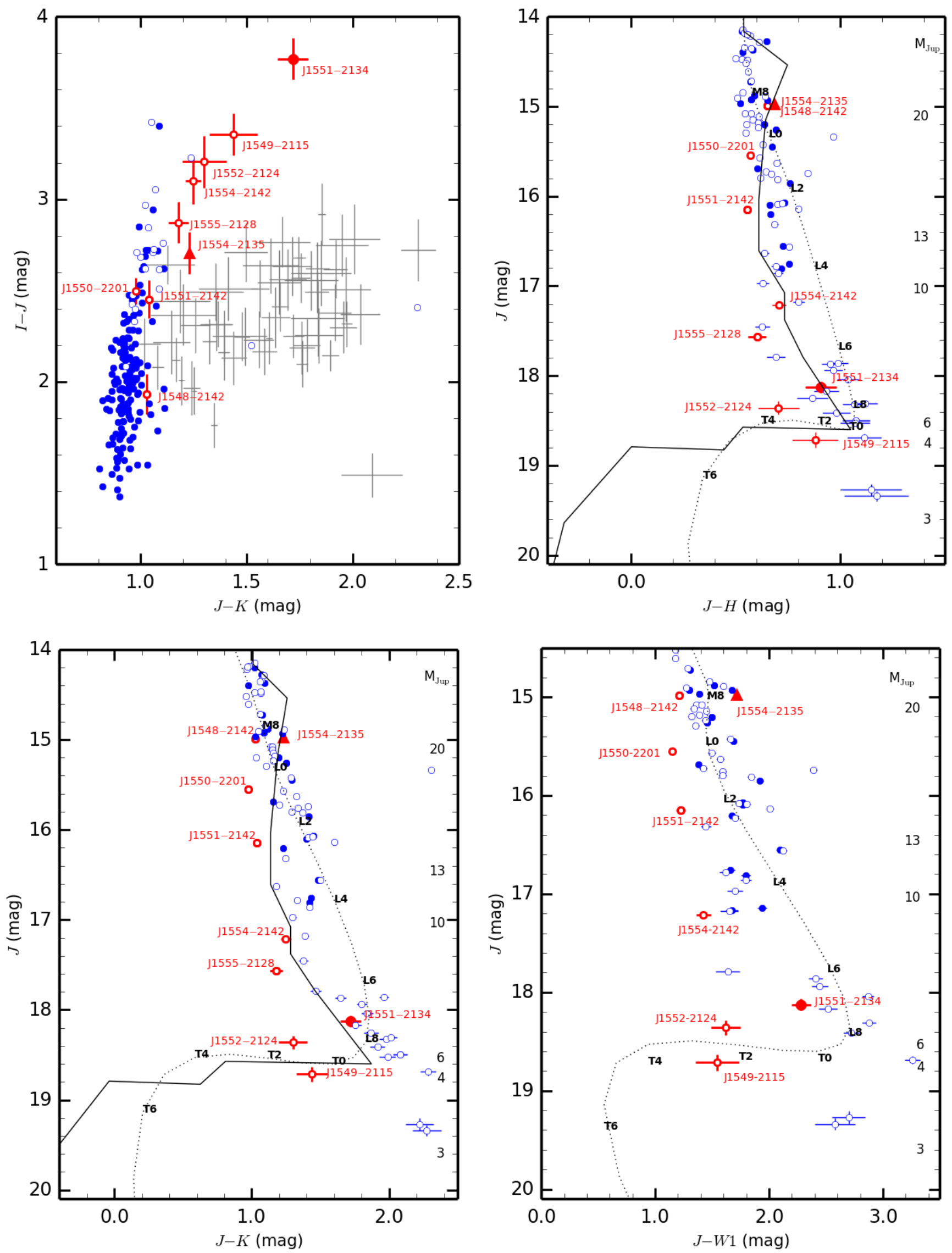

Fig. 5. Color-color (top left panel) and color-magnitude diagrams (top right and bottom panels), combining VIMOS, UKDISS, and WISE photometry. The nine $z J$ USco candidates are plotted as red symbols and are labelled. Confirmed USco members from Ardila et al. (2000) and Lodieu et al. (2011) are shown with blue solid circles, and USco candidates from Lodieu et al. (2013) are illustrated with blue open circles. Other symbols as in Fig. 3. 
allowed us to easily distinguish extended sources down to $J=$ $19 \mathrm{mag}$, as described in Sect. 3.1. The location of these objects in the $I-J$ versus $J-K$ color-color diagram of Fig. 5 diverges from that of USco members, as discussed in Sect. 3.3. We checked the FWHM of our USco candidates in all the available UKIDSS images, which confirms that they were consistent with the stellar PSFs at various explored wavelengths. To estimate the number of unresolved red galaxies that may be contaminating our VIMOSUKIDSS survey, we used the multicolor GOODS-MUSIC V2.0 catalog (Grazian et al. 2006; Santini et al. 2009) in a similar manner to Bihain et al. (2009). In the magnitude range $J=14.5-$ $20.0 \mathrm{mag}$, i.e., down to the UKIDSS $J$-band limiting magnitude, we searched for GOOD-MUSIC sources that comply with the photometric selection criteria described in Sects. 3.1 and 3.2 and found none. Furthermore, a list of USco member candidates that are free of extragalactic unresolved red contaminants can be produced by performing a proper motion analysis of the candidates (see Sect. 3.5), since very distant sources do not show significant motion at all, while USco has a distinctive proper motion.

\subsection{Proper motion analysis}

The proper motion of the young USco stellar association is $\mu_{\alpha} \cos \delta=-12.1 \pm 1.6$ and $\mu_{\delta}=-23.8 \pm 1.9$ mas yr$^{-1}$ (Zacharias et al. 2004), which is measurable using our data and data from the UKIDSS archive. The final assessment of membership in USco is done by deriving the proper motions of the seven photometric candidates. We employed various combinations of optical and near-infrared images separated in time by several years for this purpose. One collection of images was formed by UKIDSS $J$ and $K$ data (2005 June 05) and VIMOS $I$ and $z$ images (this paper), which were taken $\approx 3.8 \mathrm{yr}$ apart. Another collection of images comprised the UKIDSS $K$-band first and second epoch data (2005 June 05, 2011 March 16), providing a time baseline of $\approx 5.8 \mathrm{yr}$. For those photometric candidates fainter than $J=18.0 \mathrm{mag}$, we also used the UKIDSS $H$-band images (2005 June 05) to improve the $\mathrm{S} / \mathrm{N}$ of the astrometric measurements. The UKIDSS and VIMOS data had an average seeing of $0 .{ }^{\prime} 8$ and 0.6 , respectively.

Proper motions were obtained by comparing the target coordinates (in pixels) with the positions of $\sim 20-30$ unresolved sources within the area of $3 \times 3 \operatorname{arcmin}^{2}$, except for J1551-2134, for which a larger area of $4 \times 4 \operatorname{arcmin}^{2}$ was analyzed. Pixel transformations were derived employing third/fourth order polynomials and the GEOMAP routine within IRAF. The typical dispersion of the polynomial transformations was \pm 0.1 pix for the right ascension and declination axis after rejecting reference sources that deviated by more than 2-3 $\sigma$ from null motion, where $\sigma$ denoted the dispersion of the astrometric transformations. All of the unresolved reference sources defining the null motion were selected as having $\mathrm{S} / \mathrm{N}$ higher than 15 in the flux peak with respect to the background noise. By considering the temporal difference between images and the pixel scales and north-east orientation of the detectors, displacements in pixels were converted into the proper motion measurements provided in Table 3. The quoted astrometric uncertainties were obtained by quadratically adding the dispersions of the polynomial transformations and the errors of the targets' centroids, as provided by the automatic identification algorithms; the latter oscillated between \pm 0.01 pix for the brightest photometric candidates and \pm 0.3 pix for the faintest candidates. The astrometric error bars of the faintest targets are clearly dominated by the UKIDSS centroid errors. Table 3 also contains the time baselines, the filters, and the $\mathrm{S} / \mathrm{N}$ of the targets, as measured in the corresponding images.
Table 3. Proper motion measurements.

\begin{tabular}{|c|c|c|c|c|c|}
\hline $\begin{array}{l}\text { Abriged } \\
\text { name }\end{array}$ & $\begin{array}{c}\Delta t \\
(\mathrm{yr})\end{array}$ & $\begin{array}{l}S / N^{a} \\
1^{\mathrm{st}} / 2^{\mathrm{nd}} \\
\end{array}$ & $\begin{array}{c}\text { Images } \\
\text { UKIDSS / VIMOS } \\
\end{array}$ & $\begin{array}{c}\mu_{\alpha} \cos \delta \\
\left(\operatorname{mas~yr}^{-1}\right)\end{array}$ & $\begin{array}{c}\mu_{\delta} \\
\left(\operatorname{mas~yr}^{-1}\right)\end{array}$ \\
\hline J1554-2135 & $\begin{array}{l}3.82 \\
3.82 \\
3.82 \\
3.82 \\
5.78\end{array}$ & $\begin{array}{l}>15 />15 \\
>15 />15 \\
>15 />15 \\
>15 />15 \\
>15 />15\end{array}$ & $\begin{array}{c}J / z \\
K / z \\
J / I \\
K / I \\
K / K \\
\text { Weighted value }\end{array}$ & $\begin{array}{l}-10.61 \pm 3.69 \\
-14.01 \pm 2.89 \\
-11.19 \pm 3.73 \\
-17.97 \pm 2.22 \\
-13.03 \pm 3.07 \\
-13.72 \pm 3.73\end{array}$ & $\begin{array}{l}-20.33 \pm 3.83 \\
-21.76 \pm 3.72 \\
-17.49 \pm 3.58 \\
-13.88 \pm 2.26 \\
-22.81 \pm 3.23 \\
-20.50 \pm 3.83\end{array}$ \\
\hline J1548-2142 & $\begin{array}{l}3.87 \\
3.87 \\
3.87 \\
3.87 \\
5.78\end{array}$ & $\begin{array}{l}>15 />15 \\
>15 />15 \\
>15 />15 \\
>15 />15 \\
>15 />15\end{array}$ & $\begin{array}{c}J / z \\
K / z \\
J / I \\
K / I \\
K / K \\
\text { Weighted value }\end{array}$ & $\begin{array}{l}5.56 \pm 3.88 \\
5.53 \pm 3.11 \\
5.25 \pm 4.15 \\
5.42 \pm 3.75 \\
4.28 \pm 4.37 \\
4.75 \pm 4.37\end{array}$ & $\begin{array}{l}-14.60 \pm 4.23 \\
-17.31 \pm 3.70 \\
-14.87 \pm 4.65 \\
-15.77 \pm 3.86 \\
-16.93 \pm 3.90 \\
-16.48 \pm 4.65\end{array}$ \\
\hline $\mathrm{J} 1550-2201^{b}$ & $\begin{array}{l}3.88 \\
3.88 \\
3.88 \\
3.88 \\
5.78\end{array}$ & $\begin{array}{l}>15 />15 \\
>15 />15 \\
>15 />15 \\
>15 />15 \\
>15 />15\end{array}$ & $\begin{array}{c}J / z \\
K / z \\
J / I \\
K / I \\
K / K \\
\text { Weighted value }\end{array}$ & $\begin{array}{r}4.69 \pm 4.40 \\
-3.04 \pm 4.74 \\
13.70 \pm 5.24 \\
8.21 \pm 5.34 \\
2.76 \pm 3.49 \\
3.79 \pm 5.34\end{array}$ & $\begin{array}{l}-84.60 \pm 4.45 \\
-88.24 \pm 4.87 \\
-83.01 \pm 3.99 \\
-85.76 \pm 4.72 \\
-85.27 \pm 3.29 \\
-85.21 \pm 4.87\end{array}$ \\
\hline J1551-2142 & $\begin{array}{l}3.88 \\
3.88 \\
3.88 \\
3.88 \\
5.78\end{array}$ & $\begin{array}{l}>15 />15 \\
>15 />15 \\
>15 />15 \\
>15 />15 \\
>15 />15\end{array}$ & $\begin{array}{c}J / z \\
K / z \\
J / I \\
K / I \\
K / K \\
\text { Weighted value }\end{array}$ & $\begin{array}{r}4.81 \pm 5.42 \\
8.67 \pm 4.21 \\
5.76 \pm 5.27 \\
11.95 \pm 3.86 \\
7.15 \pm 3.69 \\
7.73 \pm 5.42\end{array}$ & $\begin{array}{l}-22.79 \pm 5.35 \\
-22.21 \pm 4.18 \\
-17.51 \pm 5.09 \\
-20.41 \pm 4.06 \\
-27.46 \pm 3.37 \\
-24.78 \pm 5.35\end{array}$ \\
\hline J1554-2142 & $\begin{array}{l}3.82 \\
3.82 \\
3.82 \\
3.82 \\
5.78\end{array}$ & $\begin{array}{l}>15 />15 \\
>15 />15 \\
>15 />15 \\
>15 />15 \\
>15 />15\end{array}$ & $\begin{array}{c}J / z \\
K / z \\
J / I \\
K / I \\
K / K \\
\text { Weighted value }\end{array}$ & $\begin{array}{l}-17.30 \pm 6.37 \\
-21.07 \pm 5.71 \\
-18.27 \pm 6.54 \\
-21.90 \pm 5.76 \\
-23.97 \pm 5.10 \\
-22.33 \pm 6.54\end{array}$ & $\begin{array}{r}10.50 \pm 6.07 \\
9.06 \pm 5.03 \\
9.48 \pm 6.47 \\
11.25 \pm 4.75 \\
10.81 \pm 4.43 \\
10.54 \pm 6.47\end{array}$ \\
\hline J1555-2128 & $\begin{array}{l}3.82 \\
3.82 \\
3.82 \\
3.82 \\
5.78\end{array}$ & $\begin{array}{l}14.7 />15 \\
>15 />15 \\
14.7 />15 \\
>15 />15 \\
>15 />15\end{array}$ & $\begin{array}{c}J / z \\
K / z \\
J / I \\
K / I \\
K / K \\
\text { Weighted value }\end{array}$ & $\begin{array}{l}-30.17 \pm 7.32 \\
-21.60 \pm 4.50 \\
-40.73 \pm 7.13 \\
-20.90 \pm 4.52 \\
-30.42 \pm 4.92 \\
-28.37 \pm 7.32\end{array}$ & $\begin{array}{r}-2.12 \pm 7.02 \\
4.27 \pm 5.34 \\
8.07 \pm 7.01 \\
-3.93 \pm 5.20 \\
-1.25 \pm 4.89 \\
-0.31 \pm 7.02\end{array}$ \\
\hline J1551-2134 & $\begin{array}{l}3.88 \\
3.88 \\
3.88 \\
3.88 \\
3.88 \\
3.88 \\
5.78\end{array}$ & $\begin{aligned} & 7.1 />15 \\
& 10.5 />15 \\
& 14.5 />15 \\
& 7.1 />15 \\
& 10.5 />15 \\
& 14.5 />15 \\
& 14.6 />15\end{aligned}$ & $\begin{array}{c}J / z \\
H / z \\
K / z \\
J / I \\
H / I \\
K / I \\
K / K \\
\text { Weighted value }\end{array}$ & $\begin{array}{r}1.66 \pm 10.82 \\
-15.07 \pm 7.07 \\
-8.73 \pm 4.68 \\
-3.22 \pm 10.90 \\
-19.88 \pm 7.03 \\
-10.84 \pm 4.27 \\
-5.68 \pm 4.85 \\
-7.76 \pm 7.07\end{array}$ & $\begin{array}{r}-26.60 \pm 11.15 \\
-9.21 \pm 6.37 \\
-13.95 \pm 4.74 \\
-27.97 \pm 11.24 \\
-12.87 \pm 6.55 \\
-16.02 \pm 4.37 \\
-22.85 \pm 5.00 \\
-19.72 \pm 6.55\end{array}$ \\
\hline $\mathrm{J} 1552-2124^{c}$ & $\begin{array}{l}3.88 \\
3.88 \\
3.88 \\
3.88 \\
3.88 \\
3.88 \\
5.78\end{array}$ & $\begin{array}{l}6.0 />15 \\
7.9 />15 \\
8.0 />15 \\
6.0 />15 \\
7.9 />15 \\
8.0 />15 \\
8.0 />15\end{array}$ & $\begin{array}{c}J / z \\
H / z \\
K / z \\
J / I \\
H / I \\
K / I \\
K / K \\
\text { Weighted value }\end{array}$ & $\begin{array}{r}-6.69 \pm 12.41 \\
-3.78 \pm 6.97 \\
9.02 \pm 6.10 \\
-5.10 \pm 12.13 \\
-5.22 \pm 6.71 \\
10.48 \pm 5.46 \\
5.23 \pm 5.38 \\
4.24 \pm 10.90\end{array}$ & $\begin{array}{r}0.37 \pm 11.16 \\
-23.15 \pm 6.68 \\
8.93 \pm 7.04 \\
-2.06 \pm 10.97 \\
-23.73 \pm 6.63 \\
9.09 \pm 6.93 \\
-0.88 \pm 6.14 \\
-3.28 \pm 11.24\end{array}$ \\
\hline $\mathrm{J} 1549-2115^{c}$ & $\begin{array}{l}3.88 \\
3.88 \\
3.88 \\
3.88 \\
3.88 \\
3.88 \\
5.78\end{array}$ & $\begin{array}{c}6.4 />15 \\
6.2 />15 \\
8.3 />15 \\
6.4 />15 \\
6.2 />15 \\
8.3 />15 \\
7.6 / 8.1\end{array}$ & $\begin{array}{c}J / z \\
H / z \\
K / z \\
J / I \\
H / I \\
K / I \\
K / K \\
\text { Weighted value }\end{array}$ & $\begin{array}{r}-20.01 \pm 12.11 \\
12.09 \pm 9.43 \\
-2.90 \pm 8.06 \\
-24.98 \pm 11.81 \\
10.82 \pm 9.31 \\
-4.75 \pm 7.96 \\
-0.11 \pm 6.22 \\
-1.10 \pm 12.11\end{array}$ & $\begin{array}{r}-12.04 \pm 10.70 \\
-9.29 \pm 9.50 \\
-0.95 \pm 8.30 \\
-14.31 \pm 10.75 \\
-10.19 \pm 8.88 \\
-7.39 \pm 7.69 \\
-3.00 \pm 6.26 \\
-5.11 \pm 10.75\end{array}$ \\
\hline
\end{tabular}

Notes. (a) $\mathrm{S} / \mathrm{N}$ of the photometric candidates on the corresponding UKIDSS and VIMOS images. ${ }^{(b)}$ Known high proper motion source (2MASS J15501151-2201213). Our proper motion measurement agrees within $1 \sigma$ with the value reported by Deacon et al. (2009) ${ }^{(c)}$ Candidates discarded in Sect. 3.3.

As shown in Table 3, for each USco candidate we managed to obtain a minimum of five proper motion measurements; all individual derivations are consistent with each other within the quoted uncertainties, except for a few cases where the $\mathrm{S} / \mathrm{N}$ of the 


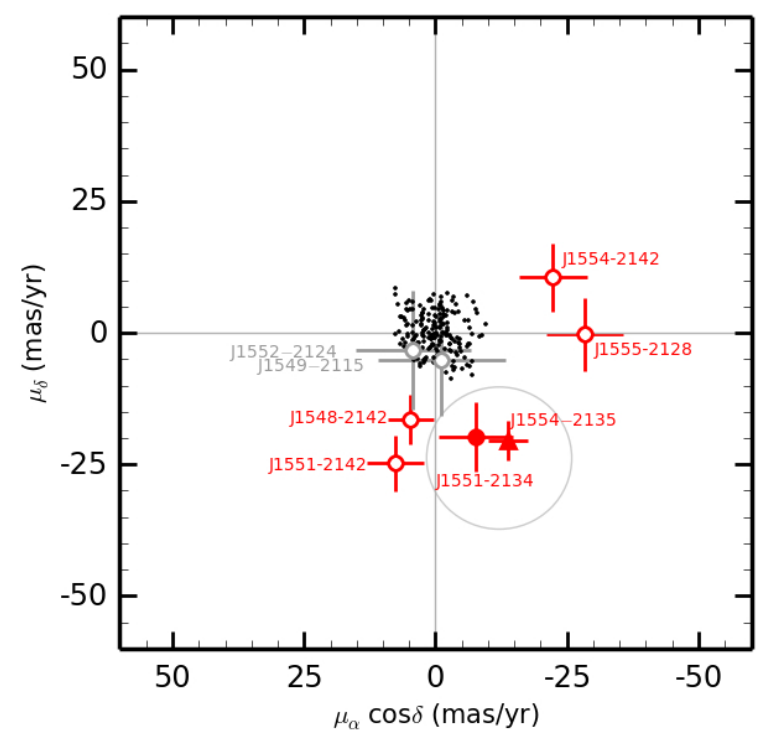

Fig. 6. Proper motion diagram of USco photometric member candidates. The seven photometric candidates are labelled and depicted in red, while the two candidates rejected in Sect. 3.3 (also labelled) are shown in gray. The gray ellipse centered at $(-12.1,-23.8)$ mas $^{-1} r^{-1}$ denotes our $2 \sigma$ astrometric criterion to identify objects with proper motion, consistent with USco membership, where $\sigma$ is the astrometric dispersion of USco low-mass members (see text). The sources used as astrometric references are presented by black dots. The high proper motion source 2MASS J15501151-2201213 (first discovered by Deacon et al. 2009) lies off limits.

target is close to the detection limit. We adopted the weighted mean motions as the final values.

Conservatively, the proper motion errors associated with the adopted motions for a given photometric candidate correspond to the largest uncertainty of the individual measurements, where the target is detected with $\mathrm{S} / \mathrm{N}$ greater than ten. Figure 6 shows the proper motion diagram, where the seven USco photometric candidates are depicted in red. The expected location of USco members is given by the ellipse of semi-major axis of $13.8 \mathrm{mas} \mathrm{yr}^{-1}$ and semi-minor axis of 13.5 mas yr centered at the motion of the young stellar association. The size of the ellipse was computed as twice the proper motion dispersions $( \pm 6.9$ mas $\mathrm{yr}^{-1}$ in $\mu_{\alpha}$, and $\pm 6.7 \mathrm{mas} \mathrm{yr}^{-1}$ in $\mu_{\delta}$ ), which were observed among the USco low-mass members and as confirmed by Lodieu et al. (2008); i.e., the ellipse defines a $2 \sigma$ criterion for the astrometric assessment of membership in USco. Five out seven photometric candidates (J1554-2142, J1555-2128, J1548-2142, J1551-2142, and J1550-2201) lie outside the $2 \sigma$ ellipse, indicating that they are not confimed members of USco by proper motion studies. The remaining two candidates have proper motions within the $2 \sigma$ ellipse of Fig. 6 , thus confirming their likely membership of USco: J1551-2134 and J1554-2135. The latter was first identified by Lodieu et al. (2008); their proper motion and our measurement agree within $0.2 \sigma$. Out of our original list of seven potential USco candidates, this number of confirmed members was expected from the level of field dwarf contamination, as discussed in Sect. 3.4.

J1551-2134 is the new likely member of USco, based on its photometry and proper motion found in the VIMOS-UKIDSS survey. Its motion deviates only by $\sim 0.3 \sigma$ with respect to the mean proper motion of the stellar association. J1551-2134 shows optical and infrared colors that agree with a mid-L spectral classification. As indicated in Table 2, there is WISE $W 1$

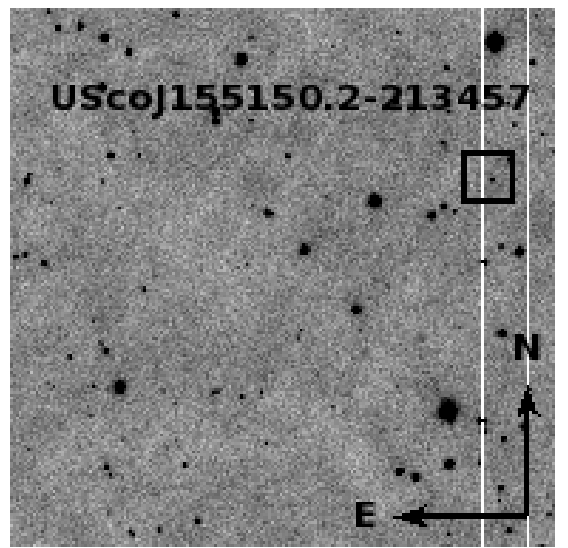

Fig. 7. Finding charts of the new USco member candidate J1551-2134. VIMOS $z$ band, $2 \times 2^{\prime}$ in size.

and $W 2$ photometry available for this object. Given the similarity between the Spitzer [4.5] and WISE W2 magnitudes for L-type dwarfs (Zapatero Osorio et al. 2011), we investigated the presence of infrared flux excesses by means of color-color diagrams, such as those presented in Fig. 5 of Peña Ramírez et al. (2012). J1551-2134 does not appear to have significant color excesses up to $4.5 \mu \mathrm{m}$. On the contrary, this object has optical and infrared colors consistent with the indices of field, high-gravity L dwarfs (Kirkpatrick et al. 2012). Figure 7 illustrates the finding chart of the new USco member.

For the sake of completeness, we also determined the proper motions of the two objects (J1552-2124 and J1549-2115) that were discarded as photometric candidates in Sect. 3.3. The measurements are given in Table 3 and are plotted as gray circles in Fig. 6. As expected, none has a proper motion compatible with the USco stellar association.

\section{Spectroscopic follow-up of J1551-2134}

The FIRE spectrum of the new photometric and astrometric likely member, J1551-2134, is presented in Fig. 8. It is compared with field, high gravity field L dwarfs in the top panel of the figure, and with young, low gravity L dwarfs in the middle panel of the figure. The comparison spectra were collected from different works: SDSS J053951.99-005902.0 and 2MASS J15150083+4847416 from Cushing et al. (2005), SIMP J2154-1055 from Gagné et al. (2014), 2MASS J22443167+2043433, GD 165B, and DENIS0205-11AB from McLean et al. (2003), and VHS J1256-1257b from Gauza et al. (2015). These data share a spectral resolution similar to the FIRE spectrum. As inferred from the top panel of Fig. 8, J1551-2134 displays a red slope that is compatible with a spectral classification of L6 with an uncertainty of one subtype. This typing agrees with the optical and infrared colors of J1551-2134.

At the age of the USco stellar association, substellar objects like J1551-2134 are expected to undergo gravitational selfcontraction. Therefore, their atmospheres are governed by conditions of low pressure and low gravity. This impacts the fine details of the output flux at cool temperatures, like those of the L types, particularly the atomic features: the lower the surface gravity, the weaker the atomic lines become (e.g., the Na I doublet at $1.14 \mu \mathrm{m}$, the two K I doublets at 1.17 and $1.25 \mu \mathrm{m}$ ). Also, the molecular features that are due to $\mathrm{FeH}$ (like the one at $1.19 \mu \mathrm{m}$, which persists down to spectral type $\sim$ L5) are affected. These and other signatures caused by low-pressure atmospheres are noticeable in the $J$-band spectrum of J1551-2134. 

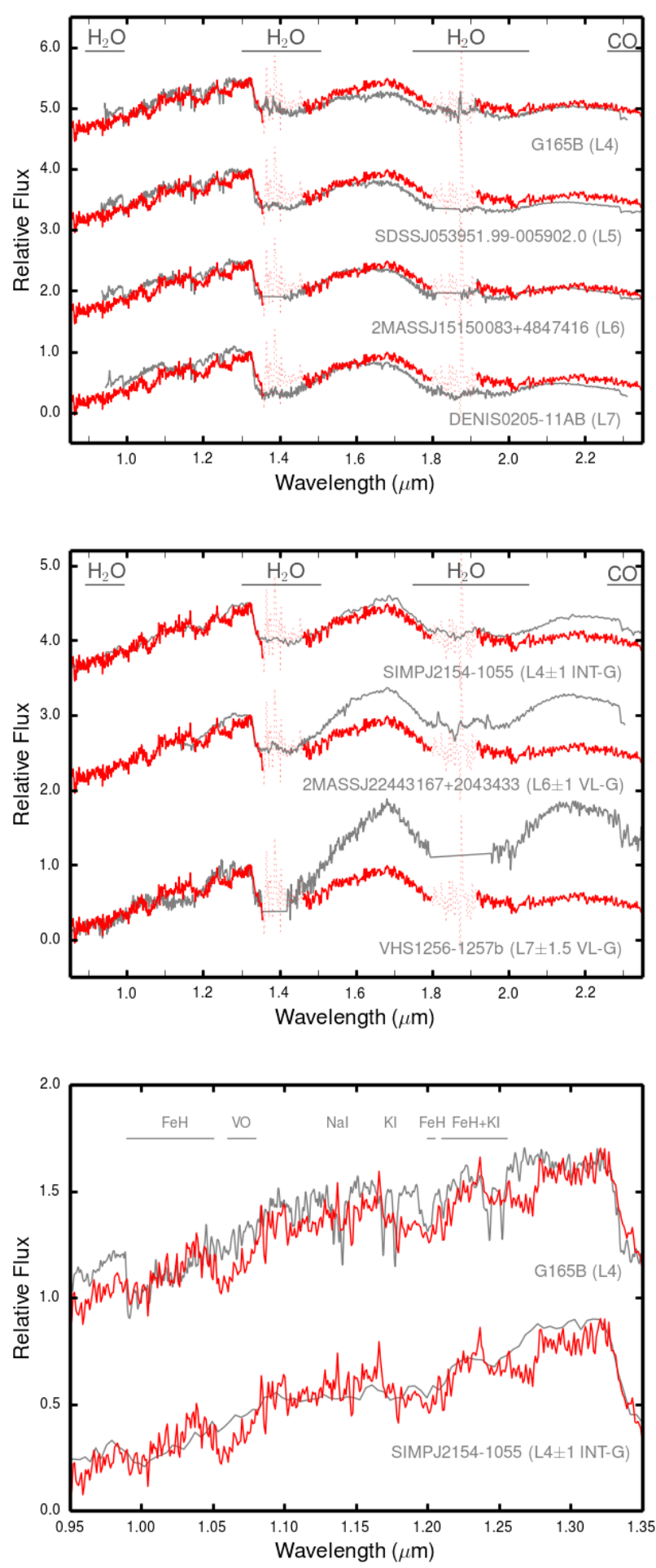

Fig. 8. FIRE low-resolution near-infrared spectrum of J1551-2134 (solid red line) compared with field high-gravity dwarf templates (top panel) and with young low- (VL-G) and intermediate-gravity (INT-G) $\mathrm{L}$ dwarfs (middle panel). The comparison spectra are labelled and plotted with solid gray lines (see text for proper references to the data). Red dotted lines depict the wavelength regions strongly affected by telluric absorption. The bottom panel illustrates the enlargement of J1551-2134's FIRE spectrum at around the K I lines ( $J$-band). All spectra are normalized to unity at $1.32 \mu \mathrm{m}$ and are shifted by a constant in the vertical direction. Some molecular and atomic features are identified.

As illustrated in the bottom panel of Fig. 8, the KI lines are not detected in J1551-2134 at the resolution of our data. We determined an upper limit on the pseudo-equivalent widths of the $J$-band $\mathrm{K}$ I lines of $\leq 6 \AA$. On the contrary, the comparison field, high-gravity dwarf (GD 165B) has strong K I absorptions. Furthermore, the 1.4-1.65 $\mu \mathrm{m} \mathrm{H}_{2} \mathrm{O}$ features of the $H$-band adopt a "triangular" shape because of low gravity atmospheres (Lucas et al. 2001; Kirkpatrick et al. 2006), a feature that is also seen in J1551-2134. Complementarily, we also measured the gravity-sensitive indices defined for low-to-intermediate resolution spectra by Allers \& Liu (2013), finding $\mathrm{FeH}_{z}=1.22 \pm 0.02$, $\mathrm{FeH}_{J}=1.16 \pm 0.03, \mathrm{KI}_{J}=0.94 \pm 0.01$ and $H$-cont $=$ $0.93 \pm 0.03$ for $\mathrm{J} 1551-2134$. All indices are compatible with low-to-intermediate gravity scores. This result, together with the upper limit on the strength of the $J$-band $\mathrm{K} I$ lines and the "triangular" shape of the $H$-band pseudo-continuum, confirm the very low-gravity nature of this object. The FIRE spectrum thus supports its membership in USco. J1551-2134 becomes a genuine young L6 \pm 1 object.

Although J1551-2134 displays clear spectral features of youth, the overall near-infrared spectral energy distribution is not similar to other low gravity L dwarfs of intermediate age (10$500 \mathrm{Myr}$ ), which show very red $J-K$ colors, like those shown in the middle panel of Fig. 8. On the contrary, the near-infrared colors and spectral slope of J1551-2134 resemble older (high gravity) field dwarfs and other similarly young mid-L dwarfs found in $\sigma$ Orionis (3 Myr) and USco (Martín et al. 2001; Lodieu et al. 2008, 2013; Peña Ramírez et al. 2012; Zapatero Osorio et al., in prep.). This suggests that the very red $J-K$ colors observed from low gravity L dwarfs of intermediate age (typically $\geq 10 \mathrm{Myr}$ ) cannot just be explained by the effects of low-gravity atmospheres, or at least the reddening of the $J-K$ color may depend nonlinearly on the low gravity. Alternative explanations, such as scenarios based on warm dusty disks/envelopes, have been proposed to reproduce the spectral behavior of very red L-type objects of intermediate age (Zapatero Osorio et al. 2010; Zakhozhay et al. 2015).

\section{Discussion}

\subsection{Masses of USco member candidates}

To estimate the masses covered by our VIMOS-UKIDSS survey, we compared the $J$-band magnitudes of our photometric candidates with the evolutionary models by the Lyon group (Baraffe et al. 1998, 2003; Chabrier et al. 2000). Theoretical luminosities and effective temperatures were converted into observables (magnitudes and colors) by applying bolometric correction-temperature and color-temperature relations given by Golimowski et al. (2004) and Hewett et al. (2006). These relations are valid for field dwarfs and we expect them to be also applicable to USco objects down to the mid-L types because of the similarity of the colors and spectral slopes between USco members and the field (see previous sections). At the age of $5 \mathrm{Myr}$ and distance of $145 \mathrm{pc}$, the substellar limit $\left(\sim 0.072 M_{\odot}\right)$ is located at about $J=13.0 \mathrm{mag}$ in the USco sequence of members, and the classical boundary between brown dwarfs and planets $\left(0.012 M_{\odot}\right.$ or $\sim 13 M_{\text {Jup }}$ ) lies at $J=16.6 \mathrm{mag}$. The VIMOS-UKIDSS survey is complete in the mass interval that goes from $0.025 M_{\odot}$ through $0.004 M_{\odot}$ (corresponding to $J=14.5-19 \mathrm{mag}$ ). Down to the limiting magnitudes, the $1.17 \mathrm{deg}^{2}$ survey would explore masses as small as $2-3 M_{\text {Jup }}$. These masses are labeled in Figs. 3-5. For the older age of $10 \mathrm{Myr}$, our combined VIMOS-UKIDSS survey would be complete in the mass interval $0.005-0.028 M_{\odot}$, reaching down to 3-4 $M_{\text {Jup }}$ at the limiting magnitudes. For either $5 \mathrm{Myr}$ or $10 \mathrm{Myr}$, our search was sensitive to the brown 
dwarf-planetary-mass object frontier and entered deeply in the planetary mass regime.

Other published surveys (Lodieu et al. 2006, 2011; Béjar et al. 2009; Dawson et al. 2013, 2014) also covered the same $1.17 \mathrm{deg}^{2}$ area, which represents a modest $0.6 \%$ of the wide USco extension. These authors mainly employ data from the DENIS, 2MASS, and UKIDSS catalogs and uncover vast extensions of this young stellar association. Béjar et al. (2009) and Lodieu et al. (2011) search for the USco population within the mass interval $\approx 0.25-0.025 M_{\odot}$, while Dawson et al. (2013, 2014) identify and study spectroscopically USco members with masses ranging from $0.09 M_{\odot}$ to $0.01 M_{\odot}$ and spectral types M5L1. Our VIMOS-UKIDSS survey, although smaller in area coverage, represents the extension towards lower planetary masses and later spectral types. The recent work by Lodieu et al. (2013), with magnitude and mass sensitivities similar to our search, is the widest and deepest search for USco photometric candidates performed to date; however, it does not include the USco region that we have studied.

As for the two photometrically, astrometrically, and spectroscopically confirmed USco members found in this work, we derived the following masses (5 Myr, $145 \mathrm{pc}$ ): $\approx 0.021 M_{\odot}(\mathrm{J} 1554-2135)$ and $\approx 8 M_{\text {Jup }}(\mathrm{J} 1551-2134$, new discovery). For an older age of $10 \mathrm{Myr}$ (see Sect. 1), the mass of the new USco member would result in $\approx 10 M_{\text {Jup. }}$ J1551-2134 appears to have a mass below the deuterium-burning mass limit even if the USco association were $10 \mathrm{Myr}$ old; it becomes one of the least massive and late-type objects known in the entire USco association.

\subsection{Mass function}

We place our findings in the context of the mass function of young clusters and other star-forming regions. Peña Ramírez et al. (2012) presented the mass function of the $3 \mathrm{Myr}$ $\sigma$ Orionis cluster that extends from the most massive stars (main sequence O-type star) all the way down to the planetary-mass domain at $4 M_{\text {Jup. }}$. These authors discuss the $\sigma$ Orionis cluster harbouring about as many brown dwarfs $\left(0.072-0.012 M_{\odot}\right)$ and planetary-mass objects $\left(0.012-0.004 M_{\odot}\right)$ as low-mass stars $\left(0.25-0.072 M_{\odot}\right)$, with a mass spectrum that smoothly increases for low masses following the expression $\Delta N / \Delta M \sim M^{-\alpha}$, where $\alpha=0.6 \pm 0.2$ for $M<0.35 M_{\odot}$. Other mass function derivations yielded rising functions with typical power-law indices between 0.4 and 1.0 in various star-forming regions: $\mathrm{ONC}, \rho \mathrm{Oph}$, NGC 2024, NGC 1333, IC 348, Cha I, Blanco 1, Pleiades, $\alpha$ Per, $\lambda$ Ori, NGC 6611, USco, Lupus 3. (See Bastian et al. 2010; Jeffries 2012; and Offner et al. 2014 for summaries; and Mužić et al. 2015, for a recent study).

Both USco and $\sigma$ Orionis likely have related solar metallicity and the two regions host massive stars, which allows us to make a direct comparison. In the VIMOS-UKIDSS area there are a total of ten known USco member candidates with masses in the range 0.18-0.025 $M_{\odot}$ (Lodieu et al. 2006, 2011; Béjar et al. 2009; Dawson et al. 2013, 2014), i.e., above the high-mass sensitivity limit of our survey. By considering the $\sigma$ Orionis mass function of Peña Ramírez et al. (2012), we predict the presence of 3.5 $5_{-1.0}^{+1.2}$ USco members (using an age of $5 \mathrm{Myr}$ ) with masses of $0.025-0.004 M_{\odot}$ equally distributed as follows: $1.7 \pm 0.4$ objects for the mass bin $0.025-0.012 M_{\odot}$ and $1.8_{-0.6}^{+0.8}$ sources that populate the least massive interval $0.012-0.004 M_{\odot}$. A similar number and distribution of objects would be expected if the USco age were 10 Myr. Despite being consistent with expectations, finding two USco members in our survey favors the low values of the $\alpha$ exponent of the power-law mass function better than the high values. What we indeed found was one USco member at each side of the brown dwarf-planetary-mass classical boundary.

As seen from comparing the USco string of members to the location of the field sequence of M-, L-, and T-type dwarfs shown in Figs. 3-5, our VIMOS-UKIDSS survey was designed deep enough to detect early-T and possibly mid-T type USco objects (this comparison did not account for the impact of low gravities on the spectral behavior of the methane atmospheres). The evolutionary models suggest that USco potential members with temperatures below $1300 \mathrm{~K}$ (the $\mathrm{L} / \mathrm{T}$ transition) and within the completeness magnitude of our survey could have masses in the interval $0.007-0.004 M_{\odot}(J=18.57-19.00 \mathrm{mag})$. The mass function of Peña Ramírez et al. (2012) predicts $\sim 0.8$ objects of this kind. We found no candidates displaying colors typical of field T dwarfs, which is compatible with predictions and allowed us to discard mass functions with $\alpha \geq 1.0,1.1$, and 1.2 with confidence levels of $90 \%, 95 \%$, and $98 \%$, respectively.

\section{Summary and conclusions}

We used deep photometric $I$ - and $z$-band data collected with the VIMOS instrument to perform a search for the least massive population of the young USco stellar association ( 5-10 Myr, $145 \mathrm{pc})$. Combined with the UKIDSS catalog, the survey explored an area of $1.17 \mathrm{deg}^{2}$ (northeast of the extense USco region) in the magnitude and mass ranges $J=14.5-19$ mag and 0.028-0.004 $M_{\odot}$ (completeness). We also employed the WISE catalog ( $W 1$ and $W 2$ magnitudes) to analyse the photometric candidates. We found an initial list of 11 photometric $z J$ candidates, which was later reduced to seven after an evaluation of the plethora of colors that cover the wavelength interval $0.8-3.4 \mu \mathrm{m}$. The proper motion study confirmed only two USco members, one of which has a brown dwarf mass of $\approx 0.020-0.022 M_{\odot}$ and was already known. The second object, J1551-2134, is a new discovery that has a planetary-mass of $\approx 0.008-0.010 M_{\odot}$ and no apparent infrared flux excesses up to $4.5 \mu \mathrm{m}$. The near-infrared spectroscopic follow-up of J1551-2134 (JHK FIRE spectrum of resolution 450 at $1.66 \mu \mathrm{m})$ confirmed the low-gravity nature of its atmosphere (weak alkaline lines, strong VO absorption, peaked $H$-band pseudocontinuum), as expected for a young cool source, and yielded a spectral type of L6 \pm 1 . J1551-2134 shows optical and infrared colors resembling those of field, high gravity dwarfs and very young $(<10 \mathrm{Myr})$ members of similar classification in marked contrast to the very red indices of field, lowgravity L dwarfs of intermediate age. This suggests that gravity alone is not the key factor when accounting for the very red nature of some young L dwarfs and/or that the colors do not depend linearly on gravity. The discovery of two USco substellar members in our VIMOS-UKIDSS survey is consistent with the low values of the exponent in the mass spectrum of $\sigma$ Orionis found by Peña Ramírez et al. (2012). The non-detection of T-type candidates in our survey allowed to constrain a mass spectrum in the interval $0.007-0.004 \mathrm{M}_{\mathrm{Jup}}, \Delta N / \Delta M \sim M^{-\alpha}$, where $\alpha<1.2$ with a confidence level of 98\%. J1551-2134 is one of the least massive and latest type members of the USco stellar association.

Acknowledgements. We thank the anonymous referee for providing us with constructive comments and suggestions. This work is based on observations made with the WHT, installed at the Spanish Observatorio del Roque de los Muchachos on the island of La Palma, Spain. This work is also based on observations made with ESO Telescopes at the Paranal Observatory under program ID 083.C-0556. This work is partly financed by the Spanish Ministry of Economy and Competitivity through the project AYA2014-54348-C3-2-R, and the Chilean FONDECYT Postdoctoral grant 3140351. 
Note added in proof. We compared the entire JHK spectrum of USco J155150.2-213457, with the data of various field young dwarfs of Allers \& Liu (2013), and Gagné et al. (2014) finding a resemblance with $\mathrm{L} 4 \pm 1$ objects, which agrees with our spectral classification. However, only the observations of young cluster and star-forming regions objects of different magnitudes will lead to the derivation of a consistent spectral sequence with decreasing temperature and luminosity.

\section{References}

Allers, K. N., \& Liu, M. C. 2013, ApJ, 772, 79

Ardila, D., Martín, E., \& Basri, G. 2000, AJ, 120, 479

Baraffe, I., Chabrier, G., Allard, F., \& Hauschildt, P. H. 1998, A\&A, 337, 403

Baraffe, I., Chabrier, G., Barman, T. S., Allard, F., \& Hauschildt, P. H. 2003, A\&A, 402, 701

Bastian, N., Covey, K. R., \& Meyer, M. R. 2010, ARA\&A, 48, 339

Béjar, V. J. S., Osorio, M. R. Z., Pérez-Garrido, A., et al. 2009, in 15th

Cambridge Workshop on Cool Stars, Stellar Systems, and the Sun, ed.

E. Stempels, AIP Conf. Ser., 1094, 469

Bihain, G., Rebolo, R., Zapatero Osorio, M. R., et al. 2009, A\&A, 506, 1169

Caballero, J. A., Burgasser, A. J., \& Klement, R. 2008, A\&A, 488, 181

Casali, M., Adamson, A., Alves de Oliveira, C., et al. 2007, A\&A, 467, 777

Chabrier, G., Baraffe, I., Allard, F., \& Hauschildt, P. 2000, ApJ, 542, 464

Costado, M. T., Béjar, V. J. S., Caballero, J. A., et al. 2005, A\&A, 443, 1021

Cushing, M. C., Rayner, J. T., \& Vacca, W. D. 2005, ApJ, 623, 1115

David, T. J., Hillenbrand, L. A., Cody, A. M., Carpenter, J. M., \& Howard, A. W. 2016, ApJ, 816, 21

Dawson, P., Scholz, A., Ray, T. P., et al. 2013, MNRAS, 429, 903

Dawson, P., Scholz, A., Ray, T. P., et al. 2014, MNRAS, 442, 1586

de Geus, E. J., de Zeeuw, P. T., \& Lub, J. 1989, A\&A, 216, 44

de Zeeuw, P. T., Hoogerwerf, R., de Bruijne, J. H. J., Brown, A. G. A., \& Blaauw,

A. 1999, AJ, 117,354

Deacon, N. R., Hambly, N. C., King, R. R., \& McCaughrean, M. J. 2009, MNRAS, 394, 857

D'Odorico, S., Aguayo, A.-M., Brillant, S., et al. 2003, The Messenger, 113, 26 Dupuy, T. J., \& Liu, M. C. 2012, ApJS, 201, 19

Epchtein, N., de Batz, B., Copet, E., et al. 1994, Ap\&SS, 217, 3

Franx, M., Labbé, I., Rudnick, G., et al. 2003, ApJ, 587, L79

Gagné, J., Lafrenière, D., Doyon, R., et al. 2014, ApJ, 792, L17

Gauza, B., Béjar, V. J. S., Pérez-Garrido, A., et al. 2015, ApJ, 804, 96

Golimowski, D. A., Leggett, S. K., Marley, M. S., et al. 2004, AJ, 127, 3516

Grazian, A., Fontana, A., de Santis, C., et al. 2006, A\&A, 449, 951

Hambly, N. C., Collins, R. S., Cross, N. J. G., et al. 2008, MNRAS, 384, 637

Herczeg, G. J., \& Hillenbrand, L. A. 2015, ApJ, 808, 23

Hewett, P. C., Warren, S. J., Leggett, S. K., \& Hodgkin, S. T. 2006, MNRAS, 367,454
Hodgkin, S. T., Irwin, M. J., Hewett, P. C., \& Warren, S. J. 2009, MNRAS, 394, 675

Jeffries, R. D. 2012, in EAS PS 57, eds. C. Reylé, C. Charbonnel, \& M. Schultheis, 45

Johnson, J. A., Marcy, G. W., Hamilton, C. M., Herbst, W., \& Johns-Krull, C. M. 2004, AJ, 128, 1265

Kirkpatrick, J. D., Barman, T. S., Burgasser, A. J., et al. 2006, ApJ, 639, 1120

Kirkpatrick, J. D., Gelino, C. R., Cushing, M. C., et al. 2012, ApJ, 753, 156

Kraus, A. L., Cody, A. M., Covey, K. R., et al. 2015, ApJ, 807, 3

Lagerholm, C., Kuntschner, H., Cappellari, M., et al. 2012, A\&A, 541, A82

Lawrence, A., Warren, S. J., Almaini, O., et al. 2007, MNRAS, 379, 1599

Leggett, S. K., Marley, M. S., Freedman, R., et al. 2007, ApJ, 667, 537

Lodieu, N., Hambly, N. C., \& Jameson, R. F. 2006, MNRAS, 373, 95

Lodieu, N., Hambly, N. C., Jameson, R. F., et al. 2007, MNRAS, 374, 372

Lodieu, N., Hambly, N. C., Jameson, R. F., \& Hodgkin, S. T. 2008, MNRAS, 383, 1385

Lodieu, N., Dobbie, P. D., \& Hambly, N. C. 2011, A\&A, 527, A24

Lodieu, N., Dobbie, P. D., Cross, N. J. G., et al. 2013, MNRAS, 435, 2474

Lucas, P. W., Roche, P. F., Allard, F., \& Hauschildt, P. H. 2001, MNRAS, 326, 695

Manchado, A., Barreto, M., Acosta-Pulido, J., et al. 2004, in SPIE Conf. Ser. 5492, eds. A. F. M. Moorwood, \& M. Iye, 1094

Martín, E. L., Zapatero Osorio, M. R., Barrado y Navascués, D., Béjar, V. J. S., \& Rebolo, R. 2001, ApJ, 558, L117

McLean, I. S., McGovern, M. R., Burgasser, A. J., et al. 2003, ApJ, 596, 561

Mužić, K., Scholz, A., Geers, V. C., \& Jayawardhana, R. 2015, ApJ, 810, 159

Offner, S. S. R., Clark, P. C., Hennebelle, P., et al. 2014, Protostars and Planets VI, 53

Patten, B. M., Stauffer, J. R., Burrows, A., et al. 2006, ApJ, 651, 502

Peña Ramírez, K., Béjar, V. J. S., Zapatero Osorio, M. R., Petr-Gotzens, M. G., \& Martín, E. L. 2012, ApJ, 754, 30

Pecaut, M. J., Mamajek, E. E., \& Bubar, E. J. 2012, ApJ, 746, 154

Preibisch, T., \& Zinnecker, H. 1999, AJ, 117, 2381

Preibisch, T., Guenther, E., Zinnecker, H., et al. 1998, A\&A, 333, 619

Preibisch, T., Brown, A. G. A., Bridges, T., Guenther, E., \& Zinnecker, H. 2002, AJ, 124, 404

Rodríguez-Ledesma, M. V., Mundt, R., Ibrahimov, M., et al. 2012, A\&A, 544, A112

Santini, P., Fontana, A., Grazian, A., et al. 2009, A\&A, 504, 751

Simcoe, R. A., Burgasser, A. J., Bernstein, R. A., et al. 2008, in SPIE Conf. Ser., 7014, 0

Simcoe, R. A., Burgasser, A. J., Schechter, P. L., et al. 2013, PASP, 125, 270

Skrutskie, M. F., Cutri, R. M., Stiening, R., et al. 2006, AJ, 131, 1163

Slesnick, C. L., Hillenbrand, L. A., \& Carpenter, J. M. 2008, ApJ, 688, 377

Zacharias, N., Urban, S. E., Zacharias, M. I., et al. 2004, AJ, 127, 3043

Zakhozhay, O., Zapatero Osorio, M. R., \& Béjar, V. J. S. 2015, MNRAS, submitted

Zapatero Osorio, M. R., Rebolo, R., Bihain, G., et al. 2010, ApJ, 715, 1408

Zapatero Osorio, M. R., Béjar, V. J. S., Goldman, B., et al. 2011, ApJ, 740, 4 